\title{
On the Optimality of Treating Interference as Noise
}

\author{
Chunhua Geng, Student Member, IEEE, Navid Naderializadeh, Student Member, IEEE, \\ Amir Salman Avestimehr, Member, IEEE, and Syed A. Jafar, Fellow, IEEE
}

\begin{abstract}
It is shown that in the $K$-user interference channel, if for each user the desired signal strength is no less than the sum of the strengths of the strongest interference from this user and the strongest interference to this user (all values in decibel scale), then the simple scheme of using point-to-point Gaussian codebooks with appropriate power levels at each transmitter and treating interference as noise (TIN) at every receiver (in short, TIN scheme) achieves all points in the capacity region to within a constant gap. The generalized degrees of freedom (GDoF) region under this condition is a polyhedron, which is shown to be fully achieved by the same scheme, without the need for time-sharing. The results are proved by first deriving a polyhedral relaxation of the GDoF region achieved by TIN, and then providing a dual characterization of this polyhedral region via the use of potential functions, and finally proving the optimality of this region in the desired regime.
\end{abstract}

Index Terms-Capacity region, Gaussian interference channel, generalized degrees of freedom (GDoF), treating interference as noise (TIN).

\section{INTRODUCTION}

$\mathbf{T}$ REATING interference as noise (TIN) when it is sufficiently weak is one of the key principles of interference management. As a robust principle that is also known to be optimal under certain conditions, TIN is interesting both from practical and theoretical perspectives.

From a practical perspective, TIN is attractive for its low complexity and robustness to channel uncertainty. TIN involves the use of only point-to-point channel codes, that are well understood, quite practical, and near-optimal in their ability to deal with unstructured noise. Further, since it requires only a coarse knowledge of the signal to interference

Manuscript received May 19, 2013; revised August 16, 2014; accepted February 9, 2015. Date of publication January 27, 2015; date of current version March 13, 2015. C. Geng and S. A. Jafar were supported in part by NSF Research under Grant CCF-1161418, Grant CCF-1319104, and Grant CCF-1317351. N. Naderializadeh and A. S. Avestimehr were supported in part by NSF CAREER under Grant 1408639, Grant CCF-1408755, Grant NETS-1419632, and Grant EARS-1411244, in part by ONR under Award N000141310094, in part by the Research Grants from Intel, Santa Clara, CA, USA, and Verizon, Delaware, OH, USA, through the 5G Project, and in part by Qualcomm, Inc., San Diego, CA, USA. This paper was presented at the 51st Allerton Conference on Communication, Control, and Computing in 2013 [1].

C. Geng and S. A. Jafar are with the Center of Pervasive Communications and Computing, Department of Electrical Engineering and Computer Science, University of California at Irvine, Irvine, CA 92697 USA (e-mail: chunhug@uci.edu; syed@eecs.uci.edu).

N. Naderializadeh and A. S. Avestimehr are with the Department of Electrical Engineering, University of Southern California, Los Angeles, CA 90089 USA (e-mail: naderial@usc.edu; avestimehr@ee.usc.edu).

Communicated by D. Guo, Associate Editor for Shannon Theory.

Color versions of one or more of the figures in this paper are available online at http://ieeexplore.iee.org.

Digital Object Identifier 10.1109/TIT.2015.2408342

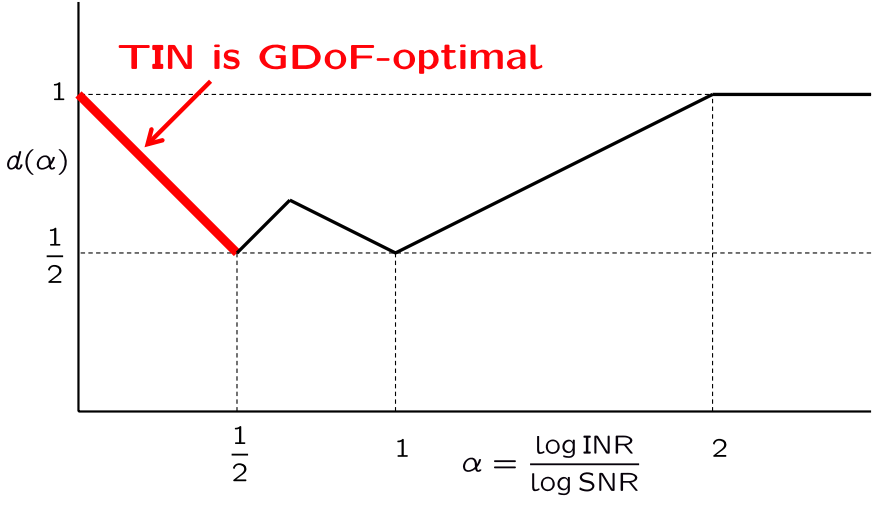

Fig. 1. The GDoF "W" curve for the two-user symmetric Gaussian interference channel.

and noise power ratio (SINR) at the transmitters, the overhead associated with acquiring channel state information at the transmitters (CSIT) is minimal for the TIN scheme. The practical appeal of the TIN scheme has motivated several studies of the achievable rate region of TIN in the literature. However, as noted by e.g., [2], [3], in spite of the simplicity of TIN, the structure of the TIN rate region is non-trivial - it involves the optimization of the power levels at the transmitters, and is generally non-convex by itself, i.e., if time-sharing is not involved.

From a theoretical perspective, it is the optimality of TIN that has attracted the most attention. It is shown in [4]-[6] that in a so-called "noisy interference" regime, TIN achieves the sum capacity of the interference channel. An extension of the noisy interference regime is obtained for multipleinput multiple-output (MIMO) Gaussian interference channels in [7]. In terms of generalized degrees of freedom (GDoF), the well-known "W" curve [8] in Fig. 1 demonstrates that for the two-user symmetric Gaussian interference channel, when the strengths of both direct channels are assumed as SNR and the interference channels are not stronger than

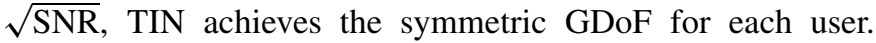
This result is generalized to $K$-user fully-connected symmetric Gaussian interference channels in [9] and to cyclic asymmetric Gaussian interference channels in [10]. However, not much is known about the regime where TIN is GDoF-optimal for the general fully-connected, fully-asymmetric $K$-user Gaussian interference channel.

In this work we present a general condition for the $K$-user fully-connected fully-asymmetric Gaussian interference channel, under which TIN is shown to be not only optimal for the entire GDoF region, but also within a constant gap of 
the entire capacity region. The general condition is stated in words as follows:

If for each user, the desired signal strength is no less than the sum of the strengths of the strongest interference from this user and the strongest interference to this user (all values in $d B$ scale), then TIN is GDoF optimal.

As an example, consider a $K$-user interference channel, where all the desired links have strength SNR and the interference links, each of which may have a different strength, are not stronger than $\sqrt{\mathrm{SNR}}$. In other words, the ratio of signal strength to the desired signal strength (each in $\mathrm{dB}$ scale) for each interferer is 0.5 or less and for the desired signal is 1. Then it follows, from the result shown in this work, that the GDoF-optimal scheme is TIN with appropriate power allocation at each transmitter.

Our proof of the optimality of TIN in the aforementioned regime consists of three steps. First, we introduce a relaxed version of TIN, called polyhedral TIN. Second, we show that, quite interestingly, the achievable GDoF region through polyhedral TIN, namely the polyhedral TIN region, can be characterized by checking the existence of a potential function for an induced fully-connected directed graph, with nodes representing the source-destination pairs in the original interference channel (with the addition of a "ground" node), and a specific assignment of lengths to each arc. Using this equivalence and a potential theorem, we derive a dual characterization of the polyhedral TIN region. The significance of our dual characterization is the elimination of power allocation variables in the characterization. Finally, we prove the outer bounds to establish the optimality of polyhedral TIN in the regime of interest. Since TIN performs no worse than polyhedral TIN, this proves that TIN is optimal in that regime in the GDoF sense.

Moreover, following the proof for GDoF-optimality of TIN, we show that in the same regime, TIN can also achieve the whole capacity region of the $K$-user interference channel to within a constant gap. Finally, we show that for general channel gain values in a $K$-user interference channel, the achievable GDoF region of TIN, namely TIN region, is composed of the union of $2^{K}$ polyhedra. However, for the regime of interest, one polyhedron subsumes all the others, hence the TIN region reduces to a single polyhedron, which is the polyhedral TIN region.

\section{System Model AND PRELIMINARIES}

As our starting point, consider the canonical model of a fully-asymmetric $K$-user wireless interference channel, with the input-output relationship

$Y_{k}(t)=\sum_{i=1}^{K} h_{k i} \tilde{X}_{i}(t)+Z_{k}(t), \quad \forall k \in[K] \triangleq\{1,2, \ldots, K\}$,

where at each time index $t, \tilde{X}_{i}(t)$ is the transmitted symbol of transmitter $i, Y_{k}(t)$ is the received signal of receiver $k$, $h_{k i}$ is the complex channel gain value from transmitter $i$ to receiver $k$, and $Z_{k}(t) \sim \mathcal{C} \mathcal{N}(0,1)$ is the additive white Gaussian noise (AWGN) at receiver $k$. All the symbols are complex. Each transmitter $i$ is subject to the power constraint $E\left[\left|\tilde{X}_{i}(t)\right|^{2}\right] \leq P_{i}$.

We will translate the standard channel model (1) into an equivalent normalized form that is more conducive for GDoF studies. We define the signal-to-noise ratio (SNR) of user $i$ and interference-to-noise ratio (INR) of transmitter $i$ at receiver $k$ as follows. ${ }^{1}$

$$
\begin{gathered}
\mathrm{SNR}_{i} \triangleq \max \left(1,\left|h_{i i}\right|^{2} P_{i}\right), \quad \mathrm{INR}_{k i} \triangleq \max \left(1,\left|h_{k i}\right|^{2} P_{i}\right), \\
i \neq k, \quad \forall i, k \in[K] .
\end{gathered}
$$

As in [8], for the GDoF metric, we preserve the ratios of different signal strengths in $\mathrm{dB}$ scale as all SNR's approach infinity. To this end, taking $P>1$ as a nominal power value, we define

$\alpha_{i i} \triangleq \frac{\log \mathrm{SNR}_{i}}{\log P}, \quad \alpha_{k i} \triangleq \frac{\log \mathrm{INR}_{k i}}{\log P}, \quad i \neq k, \quad \forall i, k \in[K]$,

implying that for each user $i, \mathrm{SNR}_{i}=P^{\alpha_{i i}}$ and for any two distinct users $i, k, \mathrm{INR}_{k i}=P^{\alpha_{k i}}$.

Now according to (2) and (3), we can represent the original channel model in (1) in the following form,

$$
Y_{k}(t)=\sum_{i=1}^{K} \sqrt{P^{\alpha_{k i}}} e^{j \theta_{k i}} X_{i}(t)+Z_{k}(t), \quad \forall k \in[K]
$$

In this equivalent channel model, $X_{i}(t)=\tilde{X}_{i}(t) / \sqrt{P_{i}}$ is the transmit symbol of transmitter $i$, and the power constraint for each transmitter is normalized to unity; i.e., $E\left[\left|X_{i}(t)\right|^{2}\right] \leq 1$, $\forall i \in[K]$. The transmit power in the original channel model is absorbed in the channel coefficients, so that $\sqrt{P^{\alpha_{k i}}}$ and $\theta_{k i}$ are the magnitude and the phase, respectively, of the channel between transmitter $i$ and receiver $k, \forall i, k \in[K]$. We will call the exponent $\alpha_{k i}$ the channel strength level of the link between transmitter $i$ and receiver $k$. In the rest of the paper, unless otherwise stated, we will consider the equivalent channel model in (4).

Since this is a $K$-user interference channel, transmitter $i$ has message $W_{i}$ intended for receiver $i$, and the messages $W_{i}$ are independent, $\forall i \in[K]$. We denote the size of the message set of user $i$ by $\left|W_{i}\right|$. For codewords spanning $n$ channel uses, the rates $R_{i}=\frac{\log \left|W_{i}\right|}{n}, i \in[K]$, are achievable if the probability of error at all the receivers can be made arbitrarily small as $n$ approaches infinity. The channel capacity region $\mathcal{C}$ is the closure of the set of all achievable rate tuples. Collecting the channel strength levels and phases in the sets

$$
\alpha \triangleq\left\{\alpha_{k i}\right\}, \quad \theta \triangleq\left\{\theta_{k i}\right\}, \quad \forall i, k \in[K]
$$

the capacity region is a function of $\alpha, \theta, P$, and is denoted as $\mathcal{C}(P, \alpha, \theta)$.

\footnotetext{
${ }^{1}$ It is not difficult to verify that assigning a value of 1 to SNR's and INR's that are less than 1 , or equivalently, assigning a 0 value to $\alpha_{i j}$ that might otherwise be negative, is only a matter of convenience, and has no impact on the GDoF or the constant gap result. The details are deferred to Appendix A.
} 


$$
\begin{aligned}
& \mathcal{D}(\alpha, \theta) \triangleq\left\{\left(d_{1}, d_{2}, \ldots, d_{K}\right): \exists\left(R_{1}(P), R_{2}(P), \ldots, R_{K}(P)\right) \in \mathcal{C}(P, \alpha, \theta) \text { as a function of } P,\right. \text { such that } \\
& \left.\qquad d_{i}=\lim _{P \rightarrow \infty} \frac{R_{i}(P)}{\log P}, \quad \forall i \in[K]\right\} .
\end{aligned}
$$

\section{A. Generalized Degrees of Freedom}

The GDoF region of the $K$-user interference channel as represented in (4) is defined in (6) at the top of the page. In general, the channel capacity (GDoF) region of complex Gaussian interference channel may depend on both the channel strength levels $\alpha$, and the channel phases $\theta$. However, the capacity (GDoF) inner and outer bounds that we present in this paper depend only on the channel strength levels $\alpha$. As such, our results hold regardless of whether or not the channel phase information is available to the transmitters.

\section{B. Capacity Region Within a Constant Gap}

Following the same definition as in [8] and [10], an achievable rate region is said to be within $x(x \geq 0)$ bits of the capacity region if for any rate tuple $\left(R_{1}, R_{2}, \ldots, R_{K}\right)$ on the boundary of the achievable rate region, the rate tuple $\left(R_{1}+x, R_{2}+x, \ldots, R_{K}+x\right)$ is outside the channel capacity region. Equivalently, $\left(\left(R_{1}-x\right)^{+},\left(R_{2}-x\right)^{+}, \ldots,\left(R_{K}-x\right)^{+}\right)$is in the achievable region for any rate tuple $\left(R_{1}, R_{2}, \ldots, R_{K}\right)$ in the capacity region, where for a real number $\beta,(\beta)^{+}$denotes $\max \{0, \beta\}$.

\section{Achievable Rate Region of TIN Scheme}

In the TIN scheme, transmitter $i$ uses a transmit power of $P^{r_{i}}, r_{i} \leq 0,{ }^{2}$ and each receiver treats all the incoming interference as noise, so that the SINR at receiver $i$ is given by

$$
\operatorname{SINR}_{i}=\frac{P^{\alpha_{i i}} \times P^{r_{i}}}{1+\sum_{j \neq i} P^{\alpha_{i j}} \times P^{r_{j}}} .
$$

This implies that the rate achieved by user $i$ through TIN is equal to

$$
R_{i}=\log \left(1+\operatorname{SINR}_{i}\right)=\log \left(1+\frac{P^{\alpha_{i i}+r_{i}}}{1+\sum_{j \neq i} P^{\alpha_{i j}+r_{j}}}\right),
$$

and therefore, the GDoF achieved by user $i$ equals

$$
d_{i}=\max \left\{0, \alpha_{i i}+r_{i}-\max \left\{0, \max _{j: j \neq i}\left(\alpha_{i j}+r_{j}\right)\right\}\right\} .
$$

The achievable GDoF region through TIN, which we denote by $\mathcal{P}^{*}$, is the set of all $K$-tuples $\left(d_{1}, d_{2}, \ldots, d_{K}\right)$ for which there exist $r_{i}$ 's, $r_{i} \leq 0, i \in[K]$, such that (8) holds for all $i \in[K]$.

\footnotetext{
${ }^{2}$ Recall that to obtain the equivalent channel model (4), in fact the transmi power of each user is absorbed into the channel coefficients. As $P$ goes to infinity, although it appears that the transmit power of user $i$ vanishes as an artifact of the GDoF framework, its actual SNR approaches infinity when the user achieves a positive GDoF value.
}

\section{CONDition For Optimality OF TIN}

The main result of this section is the following theorem, which introduces a condition under which TIN is GDoF-optimal.

Theorem 1: In a $K$-user interference channel, where the channel strength level from transmitter $i$ to receiver $j$ is equal to $\alpha_{j i}, \forall i, j \in[K]$, if the following condition is satisfied

$$
\alpha_{i i} \geq \max _{j: j \neq i}\left\{\alpha_{j i}\right\}+\max _{k: k \neq i}\left\{\alpha_{i k}\right\}, \quad \forall i, j, k \in[K],
$$

then power control and treating interference as noise can achieve the whole GDoF region. Moreover, the GDoF region is the set of all $K$-tuples $\left(d_{1}, d_{2}, \ldots, d_{K}\right)$ satisfying

$$
\begin{aligned}
0 & \leq d_{i} \leq \alpha_{i i}, \quad \forall i \in[K] \\
\sum_{j=1}^{m} d_{i_{j}} & \leq \sum_{j=1}^{m}\left(\alpha_{i_{j} i_{j}}-\alpha_{i_{j-1} i_{j}}\right), \quad \forall\left(i_{1}, i_{2}, \ldots, i_{m}\right) \in \Pi_{K}, \\
& \forall m \in\{2,3, \ldots, K\},
\end{aligned}
$$

where $\Pi_{K}$ is the set of all possible cyclic sequences ${ }^{3}$ of all subsets of $[K]$ with cardinality no less than 2 , and the modulo- $m$ arithmetic is implicitly used on the user indices, e.g., $i_{m}=i_{0}$.

Remark: Condition (9) can be stated in words as - for each user the desired signal strength is no less than the sum of the strengths of the strongest interference from this user and the strongest interference to this user (all values in $d B$ scale). Theorem 1 claims that under this condition, TIN is GDoF-optimal.

Remark: Both condition (9) and the GDoF region specified by (10)-(11) display a natural duality in the sense that they are both unchanged if the roles of the transmitters and receivers are switched, i.e., if all $\alpha_{i j}$ values are switched with $\alpha_{j i}$ values. In other words, for the same channel strengths, if we consider the reciprocal network (in the same sense as a multiple access channel being the reciprocal of a broadcast channel), then again under condition (9), TIN is GDoF-optimal, and the GDoF region is the same as in the original network. Such a duality holds also for the entire TIN region $\mathcal{P}^{*}$ (defined as the set of all $K$-tuples $\left(d_{1}, d_{2}, \ldots, d_{K}\right)$ for which there exist $r_{i}$ 's, $r_{i} \leq 0, i \in[K]$, such that (8) holds for all $i \in[K]$ ), and a similar duality relationship for the symmetric rate has been observed in [11].

Example 1: To interpret the results in Theorem 1, we derive and plot the GDoF region for a 3-user network in which condition (9) is satisfied. Consider the 3-user network in Fig. 2(a). In this network, the channel strength level between transmitter $i$ and receiver $j, \alpha_{j i}$, is shown on the corresponding link, $\forall i, j \in\{1,2,3\}$. For the case of $K=3$,

\footnotetext{
${ }^{3}$ Each cyclic sequence in $\Pi_{K}$ is essentially a cyclically ordered subset of user indices, without repetitions. In $\Pi_{K}$, there exist $\sum_{m=2}^{K}\left(\begin{array}{l}K \\ m\end{array}\right)(m-1)$ ! cyclic sequences.
} 


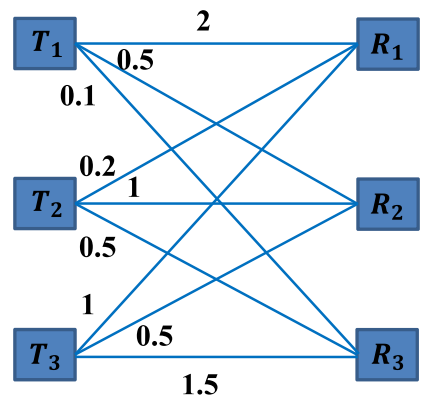

(a)

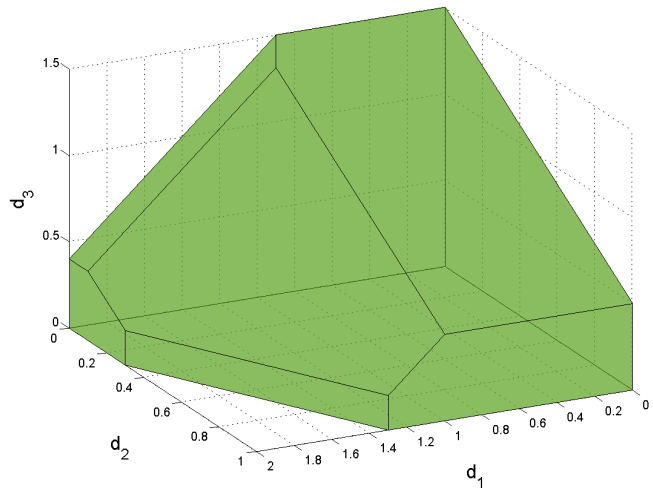

(b)

Fig. 2. (a) A 3-user interference channel, where the value on each link is equal to its channel strength level, and (b) The GDoF region of this network, which is a convex polyhedron and can be achieved by TIN.

$\Pi_{K}=\{(1,2),(1,3),(2,3),(1,2,3),(1,3,2)\}$. According to Theorem 1, the GDoF region is the set of all $\left(d_{1}, d_{2}, d_{3}\right)$ satisfying

$$
\begin{aligned}
0 \leq d_{1} & \leq 2 \\
0 \leq d_{2} & \leq 1 \\
0 \leq d_{3} & \leq 1.5 \\
d_{1}+d_{2} & \leq 2.3 \\
d_{1}+d_{3} & \leq 2.4 \\
d_{2}+d_{3} & \leq 1.5 \\
d_{1}+d_{2}+d_{3} & \leq 3.7 \\
d_{1}+d_{2}+d_{3} & \leq 2.5
\end{aligned}
$$

which is depicted in Fig. 2(b). Recall that condition (9) is satisfied in the network of Fig. 2(a) for all users $i \in\{1,2,3\}$. Therefore, Theorem 1 implies that TIN achieves the entire GDoF region of this network.

We prove Theorem 1 through the following steps. We first introduce a relaxation of the TIN scheme called polyhedral TIN, and show that the achievable GDoF region by polyhedral TIN is in fact a polyhedral region. We study the polyhedral TIN region in some detail to understand its structure. In particular, we show that the polyhedral TIN region can be characterized by checking the existence of a potential function for an induced fully-connected directed graph, with nodes representing the source-destination pairs in the original interference channel (with the addition of a "ground" node) and a specific assignment of lengths to the arcs of the graph. Afterwards, we derive a dual characterization of the polyhedral TIN region and prove the outer bounds to establish the optimality of polyhedral TIN, hence TIN, whenever condition (9) is satisfied.

\section{A. Polyhedral Relaxation of TIN}

In the first step toward proving Theorem 1, we introduce a polyhedral version of the TIN scheme. Ignoring the first $\max \{0, \ldots\}$ term in (8) changes the scheme to a relaxed version, which we call the polyhedral TIN scheme. With this modification, the achievable GDoF region via polyhedral TIN denoted by $\mathcal{P}$ will be the set of all $K$-tuples $\left(d_{1}, d_{2}, \ldots, d_{K}\right)$ for which there exist $r_{i}$ 's, $i \in[K]$, such that

$$
\begin{aligned}
r_{i} & \leq 0, \quad \forall i \in[K] \\
d_{i} & \geq 0, \quad \forall i \in[K] \\
d_{i} & =\alpha_{i i}+r_{i}-\max \left\{0, \max _{j: j \neq i}\left(\alpha_{i j}+r_{j}\right)\right\}, \quad \forall i \in[K] .
\end{aligned}
$$

In the polyhedral TIN scheme, we require that the right hand side of (14) is non-negative for all users. Otherwise, the obtained $K$-tuple is not a valid GDoF tuple and hence its corresponding power exponents $r_{i}$ 's are not acceptable for the polyhedral TIN scheme. Recall that in the original TIN scheme, for all $r_{i} \leq 0, i \in\{1,2, . ., K\}$, we always obtain a valid GDoF tuple according to (8). Therefore, this modification actually puts more constraints on the power exponents $r_{i}$ 's besides the constraints of $r_{i} \leq 0$. In general, this can only shrink the achievable GDoF region of TIN. Thus it is true that $\mathcal{P} \subseteq \mathcal{P}^{*}$.

Example 2: Consider a 2-user interference channel with $\alpha_{i j}=1, \forall i, j \in\{1,2\}$. In the polyhedral TIN scheme, since the right hand side of (14) is non-negative for all users $i \in\{1,2\}$, we have

$$
\begin{aligned}
& 1+r_{1}-\max \left\{0,1+r_{2}\right\} \geq 0 \\
& 1+r_{2}-\max \left\{0,1+r_{1}\right\} \geq 0 .
\end{aligned}
$$

Combining with the constraints of $r_{i} \leq 0$, it is easy to verify that the valid power exponents $r_{i}$ 's for the polyhedral TIN scheme satisfy $r_{1}=r_{2}$ and $r_{1}, r_{2} \in[-1,0]$, under which the polyhedral TIN region $\mathcal{P}$ only consists of the single point $(0,0)$. While in the original TIN scheme, according to (8), the achievable GDoF region $\mathcal{P}^{*}$ is the union of two line segments, i.e., $\mathcal{P}^{*}=\left\{\left(d_{1}, d_{2}\right): 0 \leq d_{1} \leq 1, d_{2}=0\right\} \cup\left\{\left(d_{1}, d_{2}\right)\right.$ : $\left.d_{1}=0,0 \leq d_{2} \leq 1\right\}$. Obviously, in this example $\mathcal{P} \subset \mathcal{P}^{*}$.

However, as we will show in the following, under condition (9), the above relaxation incurs no loss. In other words, when condition (9) is satisfied, the TIN region $\mathcal{P}^{*}$ is equal to the polyhedral TIN region $\mathcal{P}$. From (12)-(14), the polyhedral TIN region $\mathcal{P}$ can be characterized by a number of linear inequalities, which as we will see, significantly contributes to understanding the TIN region $\mathcal{P}^{*}$. In fact, it is easy to prove that $\mathcal{P}$ is the set of all $K$-tuples $\left(d_{1}, d_{2}, \ldots, d_{K}\right)$ for which there exist $r_{i}$ 's, $i \in[K]$, such that the inequalities (15)-(18) at the bottom of next page are satisfied. The details are relegated to Appendix B. 
As we will show, after the elimination of the variables $r_{i}$, $i \in[K]$, the region $\mathcal{P}$ can be fully characterized by (10)-(11). Moreover, as demonstrated in Example 1, this region is a polyhedron, which is why the scheme is called polyhedral TIN.

\section{B. Dual Characterization of Polyhedral TIN Region via Potential Functions}

Equipped with the aforementioned description of polyhedral TIN, we now characterize the polyhedral TIN region $\mathcal{P}$ for general channel strength levels. As mentioned in (15)-(18), for a GDoF tuple $\left(d_{1}, d_{2}, \ldots, d_{K}\right) \in \mathbb{R}_{+}^{K}$ (the non-negative orthant of the $K$-dimensional Euclidean space), it is in the region $\mathcal{P}$ if and only if there exist $r_{i}$ 's, $i \in[K]$, satisfying

$$
\begin{aligned}
r_{i} & \leq 0, \quad \forall i \in[K] \\
r_{i} & \geq d_{i}-\alpha_{i i}, \quad \forall i \in[K] \\
r_{i}-r_{j} & \geq \alpha_{i j}+\left(d_{i}-\alpha_{i i}\right), \quad \forall i, j \in[K], i \neq j .
\end{aligned}
$$

Now, we define a directed graph $D=(V, A)$, as shown in Fig. 3(a), where

$$
\begin{aligned}
V & =\left\{v_{1}, \ldots, v_{K}, u\right\} \\
A & =A_{1} \cup A_{2} \cup A_{3} \\
A_{1} & =\left\{\left(v_{i}, v_{j}\right): i, j \in[K], i \neq j\right\} \\
A_{2} & =\left\{\left(v_{i}, u\right): i \in[K]\right\} \\
A_{3} & =\left\{\left(u, v_{i}\right): i \in[K]\right\},
\end{aligned}
$$

and we assign a length $l(a)$ to every $\operatorname{arc} a \in A$ as follows.

$$
\begin{aligned}
l\left(v_{i}, v_{j}\right) & =\alpha_{i i}-d_{i}-\alpha_{i j} \\
l\left(v_{i}, u\right) & =\alpha_{i i}-d_{i} \\
l\left(u, v_{i}\right) & =0 .
\end{aligned}
$$

As an example, the corresponding directed graph $D$ for Example 1 is drawn in Fig. 3(b). Evidently, this is a fullyconnected directed graph, in which the length of each arc depends on the channel strength levels and the GDoFs we intend to achieve. This careful assignment of the lengths to the arcs of this graph allows us to use the following lemma.

Lemma 1: If $\mathcal{P}$ denotes the polyhedral TIN region of a $K$-user interference channel, then for a GDoF tuple $\left(d_{1}, d_{2}, \ldots, d_{K}\right) \in \mathbb{R}_{+}^{K}$, it is in the region $\mathcal{P}$ if and only if there exists a valid potential function for the graph $D$.

Proof of Lemma 1: By definition [12], a function $p: V \rightarrow \mathbb{R}$ is called a potential if for every two nodes $a, b \in V$ such that $(a, b) \in A, l(a, b) \geq p(b)-p(a)$. These inequalities only depend on the difference between potential function values. Therefore, without loss of generality, if there exists a valid potential function for the graph, we can make one node,

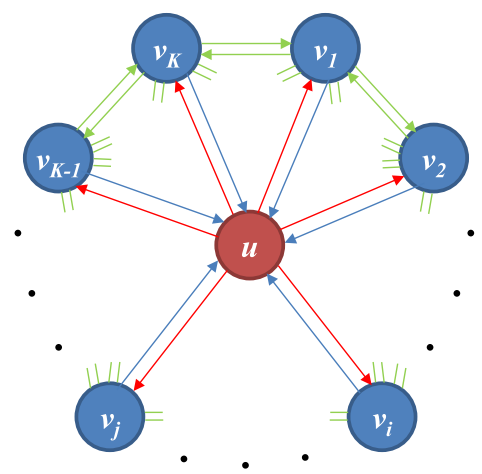

(a)

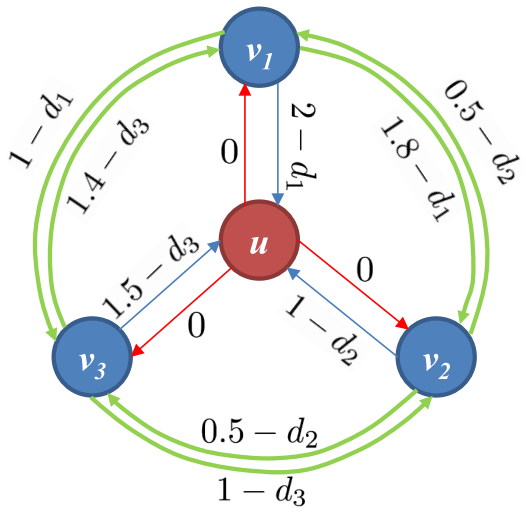

(b)

Fig. 3. (a) The directed graph $D$ in which the green, blue and red arcs belong to $A_{1}, A_{2}$ and $A_{3}$, respectively. For simplicity, only some parts of the edges are shown in this figure. (b) The corresponding directed graph $D$ for Example 1.

say node $u$, ground; i.e., $p(u)=0$. Letting $r_{i}:=p\left(v_{i}\right)$, the potential function values should satisfy the following conditions.

$$
\begin{aligned}
\alpha_{i i}-d_{i}-\alpha_{i j} & \geq r_{j}-r_{i}, \quad \forall i, j \in[K], i \neq j \\
\alpha_{i i}-d_{i} & \geq-r_{i}, \quad \forall i \in[K] \\
0 & \geq r_{i}, \quad \forall i \in[K] .
\end{aligned}
$$

The above inequalities exactly match the ones in (19)-(21). This completes the proof.

Next, we invoke the potential theorem of [12], re-stated below, to complete the characterization of the polyhedral TIN region, $\mathcal{P}$.

Potential Theorem [12, Th. 8.2]: There exists a potential function for a directed graph $D$ if and only if each directed circuit in $D$ has non-negative length.

Combining Lemma 1 and the potential theorem, we conclude that for a GDoF tuple $\left(d_{1}, d_{2}, \ldots, d_{K}\right) \in \mathbb{R}_{+}^{K}$, it is

$$
\begin{aligned}
& r_{i} \leq 0, \quad \forall i \in[K] \\
& d_{i} \geq 0, \quad \forall i \in[K] \\
& d_{i} \leq \alpha_{i i}+r_{i} \Leftrightarrow r_{i} \geq d_{i}-\alpha_{i i}, \quad \forall i \in[K] \\
& d_{i} \leq \alpha_{i i}+r_{i}-\left(\alpha_{i j}+r_{j}\right) \Leftrightarrow r_{i}-r_{j} \geq \alpha_{i j}+\left(d_{i}-\alpha_{i i}\right), \quad \forall i, j \in[K], i \neq j .
\end{aligned}
$$


in the polyhedral TIN region $\mathcal{P}$ if and only if each directed circuit in the graph $D$ has a non-negative length. Therefore, it just remains to interpret the conditions of non-negative length for the circuits.

We can categorize the circuits of $D$ in three classes:

- Circuits in the form of $\left(u, v_{i}, u\right), \forall i \in[K]$. For these circuits, we have

$$
\alpha_{i i}-d_{i} \geq 0 \Leftrightarrow d_{i} \leq \alpha_{i i} .
$$

- Circuits in the form of $\left(v_{i_{0}}, v_{i_{1}}, \ldots, v_{i_{m}}\right)$, where $i_{0}=i_{m}$, $\forall\left(i_{1}, i_{2}, \ldots, i_{m}\right) \in \Pi_{K}, \forall m \in\{2,3, \ldots, K\}$; i.e., the circuits which do not include node $u$. For these circuits, the non-negative length condition will be

$$
\begin{aligned}
& \sum_{j=0}^{m-1}\left(\alpha_{i_{j} i_{j}}-d_{i_{j}}-\alpha_{i_{j} i_{j+1}}\right) \geq 0 \\
& \Leftrightarrow \sum_{j=0}^{m-1} d_{i_{j}} \leq \sum_{j=0}^{m-1}\left(\alpha_{i_{j} i_{j}}-\alpha_{i_{j} i_{j+1}}\right) \\
& \stackrel{(a)}{\Leftrightarrow} \sum_{j=1}^{m} d_{i_{j}} \leq \sum_{j=1}^{m}\left(\alpha_{i_{j} i_{j}}-\alpha_{i_{j-1} i_{j}}\right) .
\end{aligned}
$$

where in step $(a)$ we just reorder the terms in the right hand side and use the fact that $i_{m}=i_{0}$.

- Circuits in the form of $\left(u, v_{i_{1}}, \ldots, v_{i_{m}}, u\right)$, $\forall\left(i_{1}, i_{2}, \ldots, i_{m}\right) \in \Pi_{K}, \forall m \in\{2,3, \ldots, K\}$. For these circuits, the following inequality should hold.

$$
\sum_{j=1}^{m-1}\left(\alpha_{i_{j} i_{j}}-d_{i_{j}}-\alpha_{i_{j} i_{j+1}}\right)+\left(\alpha_{i_{m} i_{m}}-d_{i_{m}}\right) \geq 0 .
$$

Since $\alpha_{i_{m} i_{1}} \geq 0$, we have $\alpha_{i_{m} i_{m}}-d_{i_{m}} \geq \alpha_{i_{m} i_{m}}-d_{i_{m}}-\alpha_{i_{m} i_{1}}$. Therefore, given the conditions in (26), the conditions in this class of circuits are redundant.

Consequently, we will end up with conditions (25)-(26). Finally adding the non-negativity constraint on $d_{i}$ 's, we obtain (10)-(11). This directly leads us to the following theorem which characterizes the polyhedral TIN region $\mathcal{P}$ for general channel strength levels.

Theorem 2: The GDoF region achieved through polyhedral TIN, denoted by $\mathcal{P}$, is the set of all $K$-tuples $\left(d_{1}, d_{2}, \ldots, d_{K}\right)$ satisfying

$$
\begin{aligned}
& 0 \leq d_{i} \leq \alpha_{i i}, \quad \forall i \in[K] \\
& \sum_{j=1}^{m} d_{i_{j}} \leq \sum_{j=1}^{m}\left(\alpha_{i_{j} i_{j}}-\alpha_{i_{j-1} i_{j}}\right), \quad \forall\left(i_{1}, i_{2}, \ldots, i_{m}\right) \in \Pi_{K}, \\
& \forall m \in\{2,3, \ldots, K\},
\end{aligned}
$$

where $\Pi_{K}$ is the set of all possible cyclic sequences of all subsets of $[K]$ with cardinality no less than 2 , and the modulo- $m$ arithmetic is implicitly used on the user indices, e.g., $i_{m}=i_{0}$.

Now, we are at a stage to complete the proof of Theorem 1.

\section{Proof of Theorem 1}

Finally, to prove Theorem 1, we will show that under condition (9), the polyhedral TIN region $\mathcal{P}$ coincides with the GDoF region outer bound, therefore establishing the optimality of TIN under (9) and proving Theorem 1. Note that from Theorem 2, the region (10)-(11) is exactly equal to the polyhedral TIN region $\mathcal{P}$ and therefore this GDoF region can be achieved by TIN. Therefore, we only need to prove the outer bounds on the GDoF region. To this end, we first present the following theorem.

Theorem 3: For the $K$-user interference channel with channel input-output relationship in (1), the capacity region is included in the set of rate tuples $\left(R_{1}, R_{2}, \ldots, R_{K}\right)$ such that

$$
\begin{aligned}
R_{i} & \leq \\
\sum_{j=1}^{m} R_{i_{j}} \leq & \sum_{j=1}^{m} \log \left(1+\left|h_{i i}\right|^{2} P_{i}\right), \quad \forall i \in[K] \\
& \left.\left.\forall\left(i_{1}, i_{2}, \ldots, i_{m}\right) \in h_{i_{j} i_{j+1}}\right|^{2} P_{i_{j+1}}+\frac{\left|h_{i_{j} i_{j}}\right|^{2} P_{i_{j}}}{1+\left|h_{i_{j-1} i_{j}}\right|^{2} P_{i_{j}}}\right), \quad \forall m \in\{2,3, \ldots, K\},
\end{aligned}
$$

where $\Pi_{K}$ is the set of all possible cyclic sequences of all subsets of $[K]$ with cardinality no less than 2 , and the modulo- $m$ arithmetic is implicitly used on the user indices, e.g., $i_{m}=i_{0}$.

The proof of Theorem 3 follows [8], [10] and is relegated to Appendix C. Equipped with Theorem 3, we can now complete the converse of Theorem 1 through the following corollary.

Corollary 1: For the $K$-user interference channel with channel input-output relationship in (4), when condition (9) is satisfied, its GDoF region is included in the set of GDoF tuples $\left(d_{1}, d_{2}, \ldots, d_{K}\right)$ such that

$$
\begin{aligned}
d_{i} & \leq \alpha_{i i}, \quad \forall i \in[K] \\
\sum_{j=1}^{m} d_{i_{j}} & \leq \sum_{j=1}^{m}\left(\alpha_{i_{j} i_{j}}-\alpha_{i_{j-1} i_{j}}\right), \quad \forall\left(i_{1}, i_{2}, \ldots, i_{m}\right) \in \Pi_{K},
\end{aligned}
$$

where $\Pi_{K}$ is the set of all possible cyclic sequences of all subsets of $[K]$ with cardinality no less than 2 , and the modulo- $m$ arithmetic is implicitly used on the user indices, e.g., $i_{m}=i_{0}$.

Proof of Corollary 1: The individual bounds in (30) follow directly from the inequalities in (28). In fact, from (28) we have

$$
d_{i}=\lim _{P \rightarrow \infty} \frac{R_{i}}{\log P} \leq \lim _{P \rightarrow \infty} \frac{\log \left(1+P^{\alpha_{i i}}\right)}{\log P}=\alpha_{i i},
$$

for any $i \in[K]$. Also, the cyclic outer bounds in (31) follow from the outer bounds in (29). In fact, for any cycle $\left(i_{1}, i_{2}, \ldots, i_{m}\right) \in \Pi_{K}$ we have

$$
\begin{aligned}
\sum_{j=1}^{m} d_{i_{j}} & =\lim _{P \rightarrow \infty} \frac{\sum_{j=1}^{m} R_{i_{j}}}{\log P} \\
& \leq \lim _{P \rightarrow \infty} \frac{\sum_{j=1}^{m} \log \left(1+P^{\alpha_{i_{j} i_{j+1}}}+\frac{P^{\alpha_{i_{j} i_{j}}}}{1+P^{\alpha_{i j} 1^{i_{j}}}}\right)}{\log P} \\
& =\sum_{j=1}^{m} \max \left\{0, \alpha_{i_{j} i_{j+1}}, \alpha_{i_{j} i_{j}}-\alpha_{i_{j-1} i_{j}}\right\} \\
& =\sum_{j=1}^{m}\left(\alpha_{i_{j} i_{j}}-\alpha_{i_{j-1} i_{j}}\right) .
\end{aligned}
$$


where the last equality is due to condition (9). This completes the proof.

Note that as we explained before, when condition (9) is satisfied, the TIN region $\mathcal{P}^{*}$ is equal to the polyhedral TIN region $\mathcal{P}$, which is a convex polyhedron as shown in Theorem 2. This means that in this regime, time-sharing cannot help enlarge the achievable GDoF region via TIN.

\section{Constant Gap to Capacity}

In this section, we show that when condition (9) holds, so that TIN is GDoF-optimal, we can apply the insight gained in the GDoF study to prove that TIN can also achieve the whole channel capacity region to within a constant gap at any finite SNR. The main result of this section is mentioned in the following theorem.

Theorem 4: In a $K$-user interference channel, where the channel strength level between transmitter $i$ and receiver $j$ is $\alpha_{j i}$, if condition (9) holds, then power control and treating interference as noise can achieve to within $\log _{2}(3 K)$ bits of the capacity region.

Proof: (Converse) Recall that using Theorem 3, we obtain the following outer bounds.

$$
\begin{aligned}
R_{i} & \leq \log _{2}\left(1+P^{\alpha_{i i}}\right), \quad \forall i \in[K] \\
\sum_{j=1}^{m} R_{i_{j}} \leq & \sum_{j=1}^{m} \log _{2}\left(1+P^{\alpha_{i_{j} i_{j+1}}}+\frac{P^{\alpha_{i_{j} i_{j}}}}{1+P^{\alpha_{i_{j-1} i_{j}}}}\right), \\
& \forall\left(i_{1}, i_{2}, \ldots, i_{m}\right) \in \Pi_{K}, \quad \forall m \in\{2,3, \ldots, K\} .
\end{aligned}
$$

Since $P>1$, it follows that

$$
\begin{aligned}
R_{i} & \leq \log _{2}\left(1+P^{\alpha_{i i}}\right) \leq \alpha_{i i} \log _{2} P+1, \quad \forall i \in[K], \\
\sum_{j=1}^{m} R_{i_{j}} & \leq \sum_{j=1}^{m} \log _{2}\left(1+P^{\alpha_{i_{j} i_{j+1}}}+\frac{P^{\alpha_{i_{j} i_{j}}}}{1+P^{\alpha_{i_{j-1} i_{j}}}}\right) \\
& <\sum_{j=1}^{m} \log _{2}\left(1+P^{\alpha_{i_{j} i_{j+1}}}+\frac{P^{\alpha_{i_{j} i_{j}}}}{P^{\alpha_{i_{j-1} i_{j}}}}\right) \\
& =\sum_{j=1}^{m} \log _{2}\left(\frac{P^{\alpha_{i_{j-1} i_{j}}}+P^{\alpha_{i_{j} i_{j+1}}+\alpha_{i_{j-1} i_{j}}}+P^{\alpha_{i_{j} i_{j}}}}{P^{\alpha_{i_{j-1} i_{j}}}}\right) \\
& \leq \sum_{j=1}^{m} \log _{2}\left(\frac{3 P^{\alpha_{i_{j} i_{j}}}}{P^{\alpha_{i_{j-1} i_{j}}}}\right) \\
& =\sum_{j=1}^{m}\left[\left(\alpha_{i_{j} i_{j}}-\alpha_{i_{j-1} i_{j}}\right) \log _{2} P+\log _{2} 3\right],
\end{aligned}
$$

for all cycles $\left(i_{1}, i_{2}, \ldots, i_{m}\right) \in \Pi_{K}, \forall m \in\{2,3, \ldots, K\}$.

(Achievability) Consider the power control and TIN scheme, where the power allocated to each transmitter is equal to $P^{r_{i}}$ $\left(r_{i} \leq 0, \forall i \in[K]\right)$, and the achievable rate for each user is

$$
R_{i, \mathrm{TIN}}=\log _{2}\left(1+\frac{P^{r_{i}+\alpha_{i i}}}{1+\sum_{j \neq i} P^{r_{j}+\alpha_{i j}}}\right) .
$$

From the proof of Theorem 1, we know that under condition (9), if $d_{i}$ 's satisfy (10) and (11), then there exist $r_{i}$ 's such that

$$
\begin{aligned}
r_{i}+\alpha_{i i}-\max _{j \neq i}\left\{0, r_{j}+\alpha_{i j}\right\} & =d_{i}, \quad \forall i, j \in[K], \\
r_{i} & \leq 0, \quad \forall i \in[K] .
\end{aligned}
$$

Therefore, we can write

$$
\begin{aligned}
R_{i, \mathrm{TIN}} & =\log _{2}\left(1+\frac{P^{r_{i}+\alpha_{i i}}}{1+\sum_{j \neq i} P^{r_{j}+\alpha_{i j}}}\right) \\
& \geq \log _{2}\left(\frac{P^{r_{i}+\alpha_{i i}}}{P^{0}+\sum_{j \neq i} P^{r_{j}+\alpha_{i j}}}\right) \\
& \geq \log _{2}\left(\frac{P^{r_{i}+\alpha_{i i}}}{K P^{r_{i}+\alpha_{i i}-d_{i}}}\right) \\
& =d_{i} \log _{2} P+\log _{2}\left(\frac{1}{K}\right) .
\end{aligned}
$$

In other words, when $d_{i}$ 's satisfy (10) and (11), the rates in (37) are always achievable by TIN, $\forall i \in[K]$. Thus it is not hard to obtain that the achievable rate region by TIN includes the rate tuples $\left(R_{1, \mathrm{TIN}}, R_{2, \mathrm{TIN}}, \ldots, R_{K, \mathrm{TIN}}\right)$ satisfying

$$
\begin{gathered}
0 \leq R_{i, \mathrm{TIN}} \leq \max \left\{0, \alpha_{i i} \log _{2} P+\log _{2}\left(\frac{1}{K}\right)\right\}, \forall i \in[K] \\
\sum_{j=1}^{m} R_{i_{j}, \mathrm{TIN}} \leq \max \left\{0, \sum_{j=1}^{m}\left[\left(\alpha_{i_{j} i_{j}}-\alpha_{i_{j-1} i_{j}}\right)\right.\right. \\
\left.\left.\times \log _{2} P+\log _{2}\left(\frac{1}{K}\right)\right]\right\}
\end{gathered}
$$

for all cycles $\left(i_{1}, i_{2}, \ldots, i_{m}\right) \in \Pi_{K}, \forall m \in\{2,3, \ldots, K\}$.

Comparing (32)-(33) with (38)-(39), we can characterize the approximate channel capacity to within a constant gap, which is only dependent on the number of users $K$. We can show that TIN achieves to within $\log _{2}(3 K)$ bits of the capacity region. To this end, we need to show that each of the rate constraints in (38) and (39) is within $\log _{2}(3 K)$ bits of its corresponding outer bound in (32) and (33); i.e., the following inequalities always hold, ${ }^{4}$

$$
\begin{aligned}
& \sigma_{R_{i}}<\log _{2}(3 K), \quad \forall i \in[K] \\
& \sigma_{\sum_{j=1}^{m} R_{i_{j}}} \leq m \log _{2}(3 K), \forall\left(i_{1}, i_{2}, \ldots, i_{m}\right) \in \Pi_{K}, \\
& \forall m \in\{2,3, \ldots, K\},
\end{aligned}
$$

where $\sigma_{(.)}$denotes the difference between the achievable rate in (38) and (39) and its corresponding outer bound in (32) and (33). For $\sigma_{R_{i}}, \forall i \in[K]$, we consider the following two cases,

- $\alpha_{i i} \log _{2} P+\log _{2}\left(\frac{1}{K}\right) \leq 0$ : In this case, we obtain

$$
\begin{aligned}
\sigma_{R_{i}} & =\alpha_{i i} \log _{2} P+1 \\
& \leq 1+\log _{2} K<\log _{2}(3 K) .
\end{aligned}
$$

\footnotetext{
${ }^{4}$ Notice that since in the second line of (33) there exists a " $<$ ", " $\leq$ " is fine for the inequality in (41)
} 
- $\alpha_{i i} \log _{2} P+\log _{2}\left(\frac{1}{K}\right)>0$ : In this case, we have

$$
\begin{aligned}
\sigma_{R_{i}} & =\left[\alpha_{i i} \log _{2} P+1\right]-\left[\alpha_{i i} \log _{2} P+\log _{2}\left(\frac{1}{K}\right)\right] \\
& =1+\log _{2} K<\log _{2}(3 K) .
\end{aligned}
$$

Similarly, for $\sigma_{\sum_{j=1}^{m} R_{i_{j}}}, \forall\left(i_{1}, i_{2}, \ldots, i_{m}\right) \in \Pi_{K}, \forall m \in$ $\{2,3, \ldots, K\}$, we consider the following two cases,

- $\sum_{j=1}^{m}\left[\left(\alpha_{i_{j} i_{j}}-\alpha_{i_{j-1} i_{j}}\right) \log _{2} P+\log _{2}\left(\frac{1}{K}\right)\right] \leq 0$ : In this case, we obtain

$$
\begin{aligned}
\sigma_{\sum_{j=1}^{m} R_{i j}} & =\sum_{j=1}^{m}\left[\left(\alpha_{i_{j} i_{j}}-\alpha_{i_{j-1} i_{j}}\right) \log _{2} P+\log _{2} 3\right] \\
& \leq \sum_{j=1}^{m}\left[\log _{2} 3+\log _{2} K\right]=m \log _{2}(3 K) .
\end{aligned}
$$

- $\sum_{j=1}^{m}\left[\left(\alpha_{i_{j} i_{j}}-\alpha_{i_{j-1} i_{j}}\right) \log _{2} P+\log _{2}\left(\frac{1}{K}\right)\right]>0$ : In this case, we have

$$
\begin{aligned}
\sigma_{\sum_{j=1}^{m} R_{i j}}= & \sum_{j=1}^{m}\left[\left(\alpha_{i_{j} i_{j}}-\alpha_{i_{j-1} i_{j}}\right) \log _{2} P+\log _{2} 3\right] \\
& -\sum_{j=1}^{m}\left[\left(\alpha_{i_{j} i_{j}}-\alpha_{i_{j-1} i_{j}}\right) \log _{2} P+\log _{2}\left(\frac{1}{K}\right)\right] \\
= & \sum_{j=1}^{m}\left[\log _{2} 3+\log _{2} K\right]=m \log _{2}(3 K) .
\end{aligned}
$$

Combining the above results, we complete the proof.

\section{The General Achievable GDof Region of TIN}

In this section, we remove the constraint (9) on the channel gains, and investigate the achievable GDoF region by TIN for $K$-user interference channels with general channel strength levels. As we show, the TIN region $\mathcal{P}^{*}$ is equal to the union of multiple polyhedra, each of which is in the form of the polyhedral TIN region of a subset of the users of the network. Remarkably, the TIN region is almost the same as the polyhedral TIN region in the sense that the measure of the difference of the two sets is zero in $\mathbb{R}^{K}$.

We have shown that when (9) holds, the original TIN region $\mathcal{P}^{*}$ is equal to the polyhedral TIN region $\mathcal{P}$. Now, the natural question to ask is what the TIN region $\mathcal{P}^{*}$ is for $K$-user interference channels with general channel strength levels. The following theorem settles this issue.

Theorem 5: In a $K$-user interference channel, where the channel strength level from transmitter $i$ to receiver $j$ is equal to $\alpha_{j i}$, the achievable GDoF region through power control and treating interference as noise, denoted by $\mathcal{P}^{*}$, is equal to

$$
\mathcal{P}^{*}=\bigcup_{\mathcal{S} \subseteq[K]} \mathcal{P}_{\mathcal{S}},
$$

where $\mathcal{P}_{\mathcal{S}}, \mathcal{S} \subseteq[K]$, is defined as

$$
\begin{array}{r}
\mathcal{P}_{\mathcal{S}}=\left\{\left(d_{1}, d_{2}, \ldots, d_{K}\right): d_{i}=0, \forall i \in \mathcal{S}, 0 \leq d_{j} \leq \alpha_{j j}, \forall j \in \mathcal{S}^{c},\right. \\
\left.\sum_{j=1}^{m} d_{i_{j}} \leq \sum_{j=1}^{m}\left(\alpha_{i_{j} i_{j}}-\alpha_{i_{j-1} i_{j}}\right), \quad \forall\left(i_{1}, i_{2}, \ldots, i_{m}\right) \in \Pi_{\mathcal{S}^{c}}\right\},
\end{array}
$$

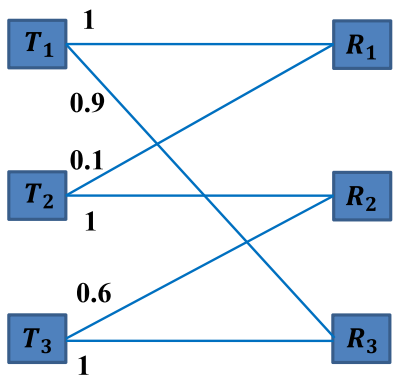

Fig. 4. A 3-user cyclic interference channel, where the channel strength level for each link is shown in the figure.

and $\Pi_{\mathcal{S}^{c}}$ is the set of all possible cyclic sequences of all subsets of $\mathcal{S}^{c}$ with cardinality no less than 2 .

The proof is given in Appendix D. In words, the TIN region $\mathcal{P}^{*}$ is the union of $2^{K}$ polyhedral TIN regions $\mathcal{P}_{\mathcal{S}}$, each of which corresponds to the case where the users in $\mathcal{S}$ are made silent. Note that $\mathcal{P}_{\phi}$ is actually the polyhedral TIN region $\mathcal{P}$ defined in Theorem 2. Except for the polyhedral TIN region $\mathcal{P}$, all the other $\mathcal{P}_{\mathcal{S}}$ 's have zero volume in $\mathbb{R}^{K}$ since in each of them the users in $\mathcal{S}$ always have zero GDoF value. Therefore, the TIN region $\mathcal{P}^{*}$ is almost the same as the polyhedral TIN region $\mathcal{P}$ in the sense that the measure of the difference of the two sets is zero in $\mathbb{R}^{K}$.

Furthermore, as opposed to the polyhedral TIN region $\mathcal{P}$, the TIN region $\mathcal{P}^{*}$ may not be convex in general, and if timesharing is allowed alongside with TIN, the achievable region may become substantially larger. Therefore, the above theorem also reveals that when the sufficient condition (9) is violated, time-sharing may help enlarge the achievable GDoF region of TIN.

Example 3: Consider the 3-user cyclic interference channel shown in Fig. 4. Notice that for user 3 the condition (9) does not hold.

First, if all the users are active, we can get the polyhedral TIN region as follows.

$$
\begin{aligned}
\mathcal{P}_{\emptyset}=\{ & \left(d_{1}, d_{2}, d_{3}\right): 0 \leq d_{i} \leq 1, \forall i \in\{1,2,3\}, \\
& d_{1}+d_{2} \leq 1.9, d_{2}+d_{3} \leq 1.4, d_{1}+d_{3} \leq 1.1, \\
& \left.d_{1}+d_{2}+d_{3} \leq 1.4\right\}
\end{aligned}
$$

which is in fact the polyhedral TIN region $\mathcal{P}$ we defined earlier.

Then, consider the cases in which only one of the three users is made silent and hence has zero GDoF, and the other two users are active. In such cases, we only need to consider the Z-channel between the remaining two users, implying that

$$
\begin{aligned}
& \mathcal{P}_{\{1\}}=\left\{\left(d_{1}, d_{2}, d_{3}\right): d_{1}=0,0 \leq d_{2} \leq 1,\right. \\
& \left.0 \leq d_{3} \leq 1, d_{2}+d_{3} \leq 1.4\right\} \\
& \mathcal{P}_{\{2\}}=\left\{\left(d_{1}, d_{2}, d_{3}\right): d_{2}=0,0 \leq d_{1} \leq 1,\right. \\
& \left.0 \leq d_{3} \leq 1, d_{1}+d_{3} \leq 1.1\right\} \\
& \mathcal{P}_{\{3\}}=\left\{\left(d_{1}, d_{2}, d_{3}\right): d_{3}=0,0 \leq d_{1} \leq 1\right. \text {, } \\
& \left.0 \leq d_{2} \leq 1, d_{1}+d_{2} \leq 1.9\right\} \text {. }
\end{aligned}
$$




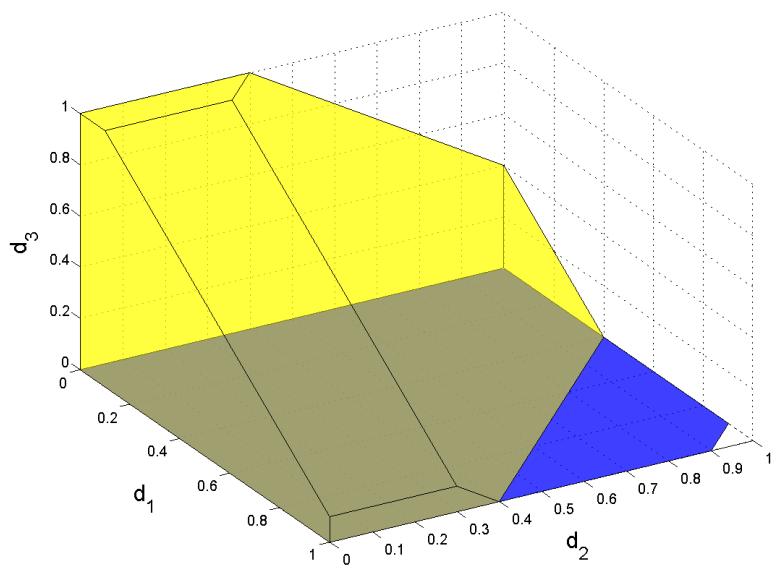

Fig. 5. The TIN region of the network in Figure 4, which is the union of the yellow region $\left(\mathcal{P}_{\emptyset}\right)$ and the blue region $\left(\mathcal{P}_{\{3\}}\right)$.

It is easy to verify that

$$
\mathcal{P}_{\{1\}} \subseteq \mathcal{P}_{\emptyset}, \mathcal{P}_{\{2\}} \subseteq \mathcal{P}_{\emptyset},
$$

but

$$
\mathcal{P}_{\{3\}} \nsubseteq \mathcal{P}_{\emptyset} .
$$

For instance, the $\operatorname{GDoF}$ tuple $(1,0.9,0) \in \mathcal{P}_{\{3\}}$ is not in the GDoF region $\mathcal{P}_{\emptyset}$ since it violates the cycle bound $d_{1}+d_{2}+d_{3} \leq 1.4$.

Next, consider the cases in which two users are made silent.

$$
\begin{aligned}
& \mathcal{P}_{\{2,3\}}=\left\{\left(d_{1}, d_{2}, d_{3}\right): 0 \leq d_{1} \leq 1, d_{2}=d_{3}=0\right\} \\
& \mathcal{P}_{\{1,3\}}=\left\{\left(d_{1}, d_{2}, d_{3}\right): 0 \leq d_{2} \leq 1, d_{1}=d_{3}=0\right\} \\
& \mathcal{P}_{\{1,2\}}=\left\{\left(d_{1}, d_{2}, d_{3}\right): 0 \leq d_{3} \leq 1, d_{1}=d_{2}=0\right\},
\end{aligned}
$$

and it can be verified that

$$
\mathcal{P}_{\{2,3\}} \subseteq \mathcal{P}_{\emptyset}, \mathcal{P}_{\{1,3\}} \subseteq \mathcal{P}_{\emptyset}, \mathcal{P}_{\{1,2\}} \subseteq \mathcal{P}_{\emptyset}
$$

Finally, we have

$$
\mathcal{P}_{\{1,2,3\}}=\left\{\left(d_{1}, d_{2}, d_{3}\right): d_{1}=d_{2}=d_{3}=0\right\} \subseteq \mathcal{P}_{\emptyset} .
$$

Therefore, the TIN region is equal to

$$
\mathcal{P}^{*}=\bigcup_{\mathcal{S} \subseteq\{1,2,3\}} \mathcal{P}_{\mathcal{S}}=\mathcal{P}_{\emptyset} \cup \mathcal{P}_{\{3\}} .
$$

This region is illustrated in Fig. 5, where the yellow region corresponds to $\mathcal{P}_{\emptyset}$ and the blue region corresponds to $\mathcal{P}_{\{3\}}$. Note that since for user 3 , the sufficient condition (9) is violated, the polyhedral TIN region $\mathcal{P}=\mathcal{P}_{\emptyset}$ is not the whole GDoF region for this 3-user cyclic channel. Moreover, as Fig. 5 shows, the region $\mathcal{P}^{*}$ is not convex. Therefore, timesharing between $\mathcal{P}_{\emptyset}$ and $\mathcal{P}_{\{3\}}$ can help enlarge the achievable GDoF region via TIN.

\section{NUMERICAL ANALYSIS}

In this section, we numerically compute the probability that the sufficient condition (9) is satisfied in a typical wireless scenario. We consider a circular cell with a radius of $1 \mathrm{~km}$ and place $K$ base stations (transmitters) randomly and uniformly over the cell area. Each base

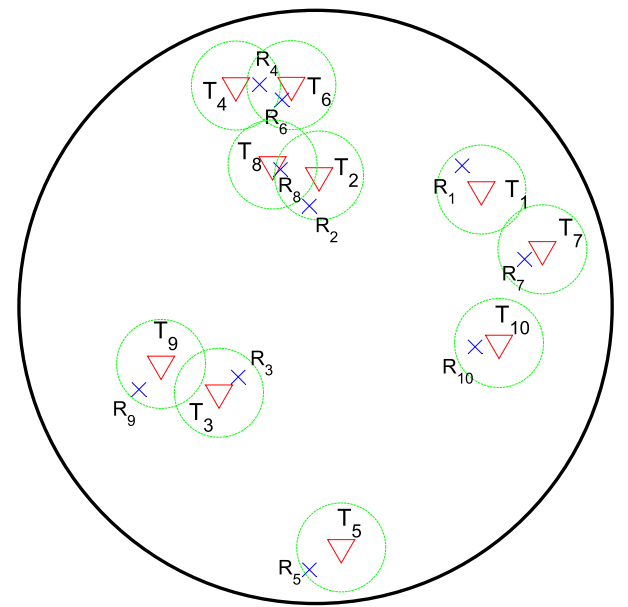

(a)

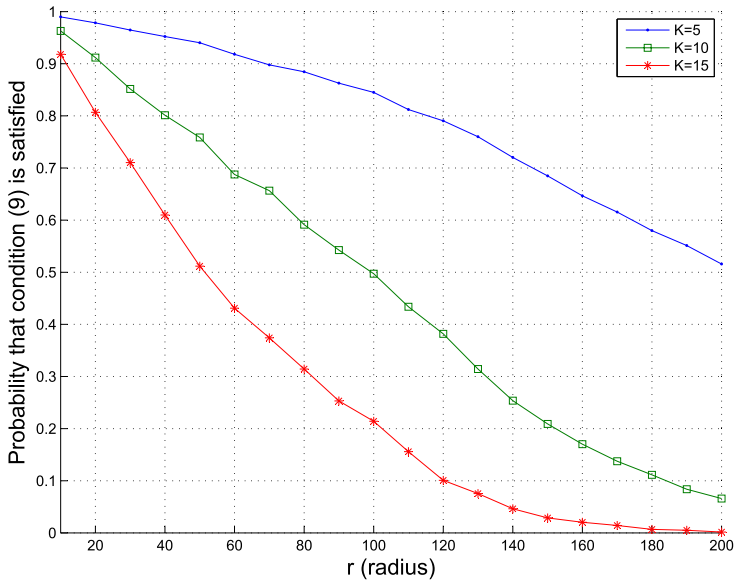

(b)

Fig. 6. (a) A 10-user interference channel where the black circle, green circles, red triangles, and blue crosses represent the whole cell area, the coverage area of base stations, base stations (transmitters), and receivers, respectively. The coverage radius of each transmitter is taken to be $r=100 \mathrm{~m}$ (b) Effect of the coverage radius and the number of users on the probability that the sufficient condition (9) is satisfied.

station is assumed to have a coverage radius of $r$. In order to create a $K$-user interference channel with strong enough direct links, we consider $K$ mobile receivers such that the $i$-th mobile receiver is located randomly and uniformly inside the coverage area of the $i$-th base station, $i \in[K]$. A realization of such a network scenario is depicted in Fig. 6(a).

For the channel gain values, we make use of the Erceg model [13], operating at a frequency of $2 \mathrm{GHz}$ and using the terrain category of hilly/light tree density. Taking the noise floor as $-110 \mathrm{dBm}$, we choose the transmit power of all the base stations such that the expected value of the SNR at the boundary of their coverage area is $0 \mathrm{~dB}$. Then, we randomly locate the base stations and mobile receivers according to the coverage radius $r$. Fig. 6(b) demonstrates the result of our numerical analysis.

As illustrated in this plot, the probability that the sufficient condition (9) for the GDoF-optimality of TIN is satisfied 


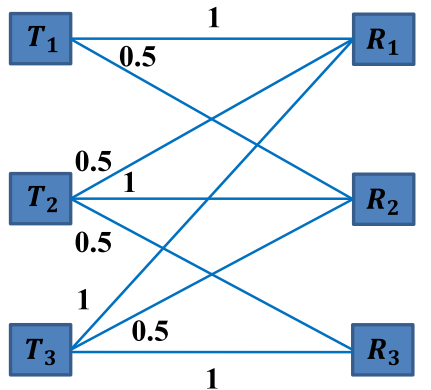

Fig. 7. A 3-user network where condition (9) is not satisfied, but TIN is still optimal. The value on each link represents its channel strength level. All channel phases are assumed to be zero.

decreases as the density of the network increases, either by increasing the number of users or by increasing the coverage radius of each base station. However, as a typical scenario, it is noteworthy that for the case of a 10-user interference channel with the coverage radius of $100 \mathrm{~m}$ for each base station, the sufficient condition (9) is satisfied half the times. This means that with a probability of $50 \%$, TIN is GDoF-optimal and can also achieve the whole capacity region of the network to within a constant gap. It therefore implies that the sufficient condition (9) can be actually satisfied in practice with a reasonably high probability, enabling the results in this paper on the optimality of treating interference as noise to be put into use in practice.

\section{COnclusion And Future Directions}

We introduced a condition for fully-connected fully-asymmetric $K$-user interference channels under which power control at the transmitters and treating interference as noise at the receivers, in short, the TIN scheme, was proven to be GDoF-optimal. The GDoF region under this condition was shown to be a polyhedron. The analysis was also generalized to show that under the same condition, TIN can achieve the whole capacity region of the network to within a constant gap that only depends on the number of users $K$. Furthermore, the achievable GDoF region by TIN for general values of channel gains in a $K$-user interference channel was also characterized fully.

In Section VI, we analyzed the probability that the aforementioned condition for the GDoF-optimality of TIN is satisfied in a typical wireless scenario. One might ask whether this condition can somehow be exploited in any general wireless network which does not satisfy this condition in its entirety. As a follow-up work, we have addressed this problem in [14] in which we showed how this condition can be utilized in any wireless network to schedule the users in a smart way and obtain considerable throughput gains over similar state-of-theart schemes.

An interesting future direction is to determine whether condition (9) is also necessary for TIN to be GDoF-optimal. For the 2-user case, it can be shown that except for a set of channel gain values of measure zero, the condition is also necessary for the GDoF-optimality of TIN. However, going beyond two users makes the problem more challenging. For instance, consider the network in Fig. 7. In this network, condition (9) is violated at users 1 and 3. However, TIN is still optimal, because it is possible to show ${ }^{5}$ that the sum-GDoF is upper bounded by 1 ; i.e.,

$$
d_{1}+d_{2}+d_{3} \leq 1 .
$$

Moreover, Theorem 2 implies that the above region is in fact the polyhedral TIN region, hence showing that TIN is optimal in the network of Fig. 7, despite the fact that condition (9) does not hold in this network. Perturbing the channel strength levels from the values in Fig. 7, or even changing the phase values if they are available to the transmitters, will invalidate the converse. We suspect that again outside a set of channel gains of measure zero, condition (9) is necessary for TIN to be GDoF-optimal. However, the necessity of this condition for the networks comprising more than 2 users remains open.

Another interesting direction following this work could be to extend the TIN scheme to include Gaussian superposition coding at the transmitters and successive interference cancellation at the receivers. In particular, it would be valuable to determine, with this additional flexibility, how much gain one can obtain beyond TIN for general channel strength levels in a $K$-user interference channel. For the case of 2-user interference channels, this problem is studied in [15], and the regimes under which such schemes outperform TIN are identified. Also, for the case of $K$-user linear deterministic interference channels, the achievable rates of such schemes are characterized as the convex hull of the feasible rates supported by the independent sets of an extended conflict graph [16]. However, general conditions for the optimality of superposition coding and successive interference cancellation are still unknown.

\section{APPENDIX A \\ REPLACING $\alpha_{i j}<0$ WITH $\alpha_{i j}=0$}

We refer to the channel with potentially negative $\alpha_{i j}$ 's $(i, j \in[K])$ as the original channel, and the channel with all negative $\alpha_{i j}$ 's replaced with zeros as the modified channel. To prove the claim that replacing $\alpha_{i j}<0$ with $\alpha_{i j}=0$ does not impact the GDoF or the constant gap result, we go through the following steps:

- First, we show that the capacity region of the original channel is within a constant gap per user to that of the modified channel, which also shows that the two channels have the same GDoF region.

The proof requires two directions, namely

$$
\mathcal{C}_{\text {original }} \subseteq \mathcal{C}_{\text {modified }}+\text { constant }
$$

and

$$
\mathcal{C}_{\text {modified }} \subseteq \mathcal{C}_{\text {original }}+\text { constant }
$$

${ }^{5}$ Note that in this network, receiver 1 , after decoding its own message and subtracting it from its received signal, has the same signal as receiver 3. Therefore, it is also able to decode the message of user 3. Besides, transmitters 1 and 3 have the same channel vectors to receivers 1 and 2 . Therefore, the sum-GDoF of this network is upper bounded by the sum-GDoF of a 2-user interference channel with $\left\{\mathrm{T}_{1,3}, \mathrm{~T}_{2}\right\}$ as the transmitters and $\left\{R_{1}, R_{2}\right\}$ as the receivers, where $T_{1,3}$ is the combination of transmitters 1 and 3 . In this 2-user interference channel, the sum-GDoF is equal to 1 [8], therefore implying that the sum-GDoF of the network in Fig. 7 is upper bounded by 1 . 
The channel input-output relationship for the original channel is given by

$$
\bar{Y}_{k}(t)=\sum_{i=1}^{K} \sqrt{P^{\bar{\alpha}_{k i}}} e^{j \theta_{k i}} X_{i}(t)+\bar{Z}_{k}(t), \quad \forall k \in[K],
$$

where $\bar{Z}_{k}(t) \sim \mathcal{C N}(0,1)$ and certain $\bar{\alpha}_{k i}$ 's might be negative. Define $\alpha_{i j} \triangleq \bar{\alpha}_{i j}^{+}, \forall i, j \in[K]$, where for any real number $\beta, \beta^{+}$stands for $\max \{0, \beta\}$. The received signal of user $k, k \in[K]$, in the modified channel is

$$
\begin{aligned}
Y_{k}(t) & =\sum_{i=1}^{K} \sqrt{P^{\alpha_{k i}}} e^{j \theta_{k i}} X_{i}(t)+Z_{k}(t) \\
& =\sum_{i=1}^{K} \sqrt{P^{\bar{\alpha}_{k i}^{+}}} e^{j \theta_{k i}} X_{i}(t)+Z_{k}(t) \\
& =\sum_{i \in \mathcal{N}_{k}} e^{j \theta_{k i}} X_{i}(t)+\sum_{i \notin \mathcal{N}_{k}} \sqrt{P^{\bar{\alpha}_{k i}}} e^{j \theta_{k i}} X_{i}(t)+Z_{k}(t),
\end{aligned}
$$

where $Z_{k}(t) \sim \mathcal{C N}(0,1)$ is independent of $\bar{Z}_{k}(t), \mathcal{N}_{k}$ is the set of transmitter indices whose link to receiver $k$ is with negative channel strength level in the original channel; i.e.,

$$
\mathcal{N}_{k}=\left\{i \in[K]: \bar{\alpha}_{k i}<0\right\} .
$$

First, we prove $\mathcal{C}_{\text {original }} \subseteq \mathcal{C}_{\text {modified }}+$ constant. Define $\mathcal{W} \triangleq\left\{W_{1}, W_{2}, \ldots, W_{K}\right\}$, and let

$$
\begin{aligned}
\hat{Y}_{k}(t) & =\bar{Y}_{k}(t)-Y_{k}(t) \\
& =\sum_{i \in \mathcal{N}_{k}}\left(\sqrt{P^{\bar{\alpha}_{k i}}}-1\right) e^{j \theta_{k i}} X_{i}(t)+\bar{Z}_{k}(t)-Z_{k}(t) .
\end{aligned}
$$

Then, we have

$$
\begin{aligned}
& I\left(W_{k} ; \bar{Y}_{k}^{n}\right) \\
& \quad \leq I\left(W_{k} ; Y_{k}^{n}, \hat{Y}_{k}^{n}\right) \\
& =I\left(W_{k} ; Y_{k}^{n}\right)+I\left(W_{k} ; \hat{Y}_{k}^{n} \mid Y_{k}^{n}\right) \\
& =I\left(W_{k} ; Y_{k}^{n}\right)+h\left(\hat{Y}_{k}^{n} \mid Y_{k}^{n}\right)-h\left(\hat{Y}_{k}^{n} \mid Y_{k}^{n}, W_{k}\right) \\
& \stackrel{(a)}{\leq} I\left(W_{k} ; Y_{k}^{n}\right)+h\left(\hat{Y}_{k}^{n}\right)-h\left(\hat{Y}_{k}^{n} \mid Y_{k}^{n}, \mathcal{W}\right) \\
& =I\left(W_{k} ; Y_{k}^{n}\right)+h\left(\hat{Y}_{k}^{n}\right)-h\left(\bar{Z}_{k}^{n}-Z_{k}^{n} \mid \mathcal{W}, Z_{k}^{n}\right) \\
& \stackrel{(b)}{\leq} I\left(W_{k} ; Y_{k}^{n}\right)+\sum_{t=1}^{n} h\left(\hat{Y}_{k}(t)\right)-h\left(\bar{Z}_{k}^{n}\right) \\
& \stackrel{(c)}{\leq} I\left(W_{k} ; Y_{k}^{n}\right)+n \log [\pi e(K+2)]-n \log (\pi e) \\
& =I\left(W_{k} ; Y_{k}^{n}\right)+n \log (K+2),
\end{aligned}
$$

where step (a) follows the facts that dropping conditioning does not reduce entropy (for the second term) and adding conditioning does not increase entropy (for the third term), step (b) follows the chain rule and the fact that dropping conditioning does not reduce entropy, and step (c) holds since $\left|\mathcal{N}_{k}\right| \leq K$ and Gaussian distribution maximizes differential entropy under a given variance constraint. This implies that
$\mathcal{C}_{\text {original }} \subseteq \mathcal{C}_{\text {modified }}+$ constant. Similarly, we can prove the other direction $\mathcal{C}_{\text {modified }} \subseteq \mathcal{C}_{\text {original }}+$ constant,

$$
\begin{aligned}
& I\left(W_{k} ; Y_{k}^{n}\right) \\
& \quad \leq I\left(W_{k} ; \bar{Y}_{k}^{n}, \hat{Y}_{k}^{n}\right) \\
& \quad=I\left(W_{k} ; \bar{Y}_{k}^{n}\right)+I\left(W_{k} ; \hat{Y}_{k}^{n} \mid \bar{Y}_{k}^{n}\right) \\
& =I\left(W_{k} ; \bar{Y}_{k}^{n}\right)+h\left(\hat{Y}_{k}^{n} \mid \bar{Y}_{k}^{n}\right)-h\left(\hat{Y}_{k}^{n} \mid \bar{Y}_{k}^{n}, W_{k}\right) \\
& \leq I\left(W_{k} ; \bar{Y}_{k}^{n}\right)+h\left(\hat{Y}_{k}^{n}\right)-h\left(\hat{Y}_{k}^{n} \mid \bar{Y}_{k}^{n}, \mathcal{W}\right) \\
& =I\left(W_{k} ; \bar{Y}_{k}^{n}\right)+h\left(\hat{Y}_{k}^{n}\right)-h\left(\bar{Z}_{k}^{n}-Z_{k}^{n} \mid \mathcal{W}, \bar{Z}_{k}^{n}\right) \\
& \leq I\left(W_{k} ; \bar{Y}_{k}^{n}\right)+\sum_{t=1}^{n} h\left(\hat{Y}_{k}(t)\right)-h\left(Z_{k}^{n}\right) \\
& \leq I\left(W_{k} ; \bar{Y}_{k}^{n}\right)+n \log (K+2) .
\end{aligned}
$$

- Next, we prove that, regardless of whether or not TIN is GDoF-optimal, the original and modified channels always have the same achievable GDoF region through TIN $\mathcal{P}^{*}$. To this end, we only need to show that using the same transmit power vector $\left(P^{r_{1}}, P^{r_{2}}, \ldots, P^{r_{K}}\right)$ in the modified and original channels, user $i \in[K]$ in both channels achieves the same GDoF value by treating interference as noise.

Recall that in the modified channel, when each transmitter $i$ uses a transmit power of $P^{r_{i}}, r_{i} \leq 0$ and each receiver treats all the incoming interference as noise, the rate achieved by user $i$ is

$$
R_{i}=\log \left(1+\frac{P^{\alpha_{i i}+r_{i}}}{1+\sum_{j \neq i} P^{\alpha_{i j}+r_{j}}}\right),
$$

and the achievable GDoF by user $i$ through TIN equals

$$
d_{i}=\max \left\{0, \alpha_{i i}+r_{i}-\max \left\{0, \max _{j: j \neq i}\left(\alpha_{i j}+r_{j}\right)\right\}\right\} .
$$

Now consider the original channel. Similarly, applying the same transmit power $P^{r_{i}}$ to each transmitter $i$ and treating interference as noise at each receiver, the achievable rate of user $i$ is

$$
\bar{R}_{i}=\log \left(1+\frac{P^{\bar{\alpha}_{i i}+r_{i}}}{1+\sum_{j \neq i} P^{\bar{\alpha}_{i j}+r_{j}}}\right) .
$$

In the original channel, denote the set of user indices whose direct link is with negative channel strength level as $\mathcal{U}$. For all the users $i \in \mathcal{U}$, it is easy to verify that the achievable GDoF through TIN is

$$
\bar{d}_{i}=0
$$

while for the users $i \notin \mathcal{U}$, we have

$$
\begin{aligned}
\bar{d}_{i} & =\max \left\{0, \bar{\alpha}_{i i}+r_{i}-\max \left\{0, \max _{j: j \neq i}\left(\bar{\alpha}_{i j}+r_{j}\right)\right\}\right\} \\
& \stackrel{(d)}{=} \max \left\{0, \bar{\alpha}_{i i}^{+}+r_{i}-\max \left\{0, \max _{j: j \neq i}\left(\bar{\alpha}_{i j}+r_{j}\right)\right\}\right\} \\
& \stackrel{(e)}{=} \max \left\{0, \bar{\alpha}_{i i}^{+}+r_{i}-\max \left\{0, \max _{j: j \neq i}\left(\bar{\alpha}_{i j}^{+}+r_{j}\right)\right\}\right\} \\
& =\max \left\{0, \alpha_{i i}+r_{i}-\max \left\{0, \max _{j: j \neq i}\left(\alpha_{i j}+r_{j}\right)\right\}\right\},
\end{aligned}
$$


where step $(d)$ follows from the fact that $\bar{\alpha}_{i i}^{+}=\bar{\alpha}_{i i}$ for users $i \notin \mathcal{U}$, and step $(e)$ holds since when $\bar{\alpha}_{i j}<0$, we have $\bar{\alpha}_{i j}+r_{j}<0, \bar{\alpha}_{i j}^{+}+r_{j} \leq 0$, and replacing the former with the latter does not impact the final result.

Combining (46) and (47), we obtain that for user $i \in[K]$

$$
\bar{d}_{i}=\max \left\{0, \alpha_{i i}+r_{i}-\max \left\{0, \max _{j: j \neq i}\left(\alpha_{i j}+r_{j}\right)\right\}\right\}
$$

Comparing (45) with (48), we establish that the original and modified channels have the same TIN region $\mathcal{P}^{*}$.

- Finally, we show that for the original channel, TIN achieves the capacity region to within $\log _{2}(3 K)$ bits, when the following condition holds

$$
\bar{\alpha}_{i i}^{+} \geq \max _{j: j \neq i}\left\{\bar{\alpha}_{j i}^{+}\right\}+\max _{k: k \neq i}\left\{\bar{\alpha}_{i k}^{+}\right\}, \quad \forall i \in[K] .
$$

We start with the converse. For the original channel, when condition (49) holds, based on Theorem 3, we have

$$
\begin{aligned}
& R_{i} \leq \log _{2}\left(1+P^{\bar{\alpha}_{i i}}\right) \leq \log _{2}\left(1+P^{\bar{\alpha}_{i i}^{+}}\right) \\
& \leq \bar{\alpha}_{i i}^{+} \log _{2} P+1=\alpha_{i i} \log _{2} P+1, \forall i \in[K] \\
& \sum_{j=1}^{m} R_{i_{j}} \\
& \leq \sum_{j=1}^{m} \log _{2}\left(1+P^{\bar{\alpha}_{i_{j} i_{j+1}}}+\frac{P^{\bar{\alpha}_{i_{j} i_{j}}}}{1+P^{\bar{\alpha}_{i_{j-1} i_{j}}}}\right) \\
& \leq \sum_{j=1}^{m} \log _{2}\left(1+P^{\bar{\alpha}_{i_{j} i_{j+1}}}+\frac{P^{\bar{\alpha}_{i_{j} i_{j}}^{+}}}{1+P^{\bar{\alpha}_{i_{j-1} i_{j}}}}\right) \\
& =\sum_{j=1}^{m} \log _{2}\left(1+P^{\bar{\alpha}_{i_{j} i_{j+1}}}+\frac{P^{\bar{\alpha}_{i_{i} i_{j}}^{+}}}{P^{0}+P^{\bar{\alpha}_{i j-1} i_{j}}}\right) \\
& <\sum_{j=1}^{m} \log _{2}\left(1+P^{\bar{\alpha}_{i_{j} i_{j+1}}}+\frac{P^{\bar{\alpha}_{i_{j} i_{j}}^{+}}}{P^{\bar{\alpha}_{i_{j-1} i_{j}}^{+}}}\right) \\
& =\sum_{j=1}^{m} \log _{2}\left(\frac{P^{\bar{\alpha}_{i_{j-1} i_{j}}^{+}}+P^{\bar{\alpha}_{i_{j} i_{j+1}}+\bar{\alpha}_{i_{j-1} i_{j}}^{+}}+P^{\bar{\alpha}_{i_{j} i_{j}}^{+}}}{P^{\bar{\alpha}_{i_{j-1}}^{+} i_{j}}}\right) \\
& \leq \sum_{j=1}^{m} \log _{2}\left(\frac{3 P^{\bar{\alpha}_{i_{j} i_{j}}^{+}}}{P^{\bar{\alpha}_{i_{j-1} i_{j}}^{+}}}\right) \\
& =\sum_{j=1}^{m}\left[\left(\bar{\alpha}_{i_{j} i_{j}}^{+}-\bar{\alpha}_{i_{j-1} i_{j}}^{+}\right) \log _{2} P+\log _{2} 3\right] \\
& =\sum_{j=1}^{m}\left[\left(\alpha_{i_{j} i_{j}}-\alpha_{i_{j-1} i_{j}}\right) \log _{2} P+\log _{2} 3\right],
\end{aligned}
$$

to within $\log _{2}(3 K)$ bits of the capacity region.

Combining the above steps, we prove that assigning a 0 value to negative $\alpha_{i j}, \forall i, j \in[K]$, has no impact on the GDoF or the constant gap results presented in this paper (i.e., Theorems 1, 4 and 5).

\section{APPENDIX B}

\section{Characterization of THE Polyhedral TIN Region $\mathcal{P}$}

To prove the equivalence of the two representations (12)-(14) and (15)-(18), we first define that $\overline{\mathcal{P}}$ is the set of all $K$-tuples $\left(d_{1}, d_{2}, \ldots, d_{K}\right)$ for which there exist $r_{i}$ 's, $i \in[K]$, such that (15)-(18) hold, and then show that $\mathcal{P}=\overline{\mathcal{P}}$.

Obviously, it is true that $\mathcal{P} \subseteq \overline{\mathcal{P}}$. This is because if $\left(d_{1}, d_{2}, \ldots, d_{K}\right) \in \mathcal{P}$, then there exist $r_{i}$ 's such that (12)-(14) are satisfied, which in turn implies that (15)-(18) are satisfied. This leads to the fact that $\left(d_{1}, d_{2}, \ldots, d_{K}\right) \in \overline{\mathcal{P}}$. Also, it is easily verified that $\overline{\mathcal{P}} \subseteq \mathcal{P}$. This is because when $\left(d_{1}, d_{2}, \ldots, d_{K}\right) \in \overline{\mathcal{P}}$, there exist $r_{i}$ 's such that (15)-(18) are satisfied, implying that

$$
\begin{aligned}
d_{i} & \leq \alpha_{i i}+r_{i}-\max \left\{0, \max _{j: j \neq i}\left(\alpha_{i j}+r_{j}\right)\right\} \\
\Leftrightarrow & r_{i} \geq d_{i}-\alpha_{i i}+\max \left\{0, \max _{j: j \neq i}\left(\alpha_{i j}+r_{j}\right)\right\}, \quad \forall i \in[K] .
\end{aligned}
$$

Now, to prove $\left(d_{1}, d_{2}, \ldots, d_{K}\right) \in \mathcal{P}$, we need to show that when (15)-(18) hold, there exist $\bar{r}_{i}$ 's such that

$$
\begin{aligned}
& \bar{r}_{i} \leq 0, \quad \forall i \in[K], \\
& \bar{r}_{i}=d_{i}-\alpha_{i i}+\max \left\{0, \max _{j: j \neq i}\left(\alpha_{i j}+\bar{r}_{j}\right)\right\}, \quad \forall i \in[K] .
\end{aligned}
$$

Denote $\mathbf{r}=\left(r_{1}, r_{2}, \ldots, r_{K}\right)$ and $\overline{\mathbf{r}}=\left(\bar{r}_{1}, \bar{r}_{2}, \ldots, \bar{r}_{K}\right)$. Writing (53) and (54) in the vector form, we have

$$
\begin{aligned}
& \overline{\mathbf{r}} \leq 0, \\
& \overline{\mathbf{r}}=f(\overline{\mathbf{r}}) .
\end{aligned}
$$

Then it is easy to verify the following conditions are satisfied: (i) $f$ is a continuous increasing function; (ii) From (52), the set $\mathcal{S}_{\overline{\mathbf{r}}}=\{\overline{\mathbf{r}}: \overline{\mathbf{r}} \geq f(\overline{\mathbf{r}})\}$ is nonempty;
Theorem 1) as $\mathcal{R}_{\text {TIN }}$. In the modified channel, for any rate tuple $R_{\mathrm{TIN}}=\left(R_{1}, R_{2}, \ldots, R_{K}\right)$ in the achievable power vector $P_{\mathrm{TIN}}=\left(P^{r_{1}}, P^{r_{2}}, \ldots, P^{r_{K}}\right)$. We denote the in $\mathcal{R}_{\text {TIN }}$ as $\mathcal{P}_{\text {TIN }}$. In the original channel, applying the and treating interference as noise at each receiver, one (i) any user $k \notin \mathcal{U}$ achieves a rate no less than that vector is utilized, as for that user the interfering links modified channel, which indicates that for users $k \notin \mathcal{U}$ the constant gap cannot increase in the original channel; 1 bit, since according to (50) the achievable rate of that with the constant gap result for the modified channel inal . . . 


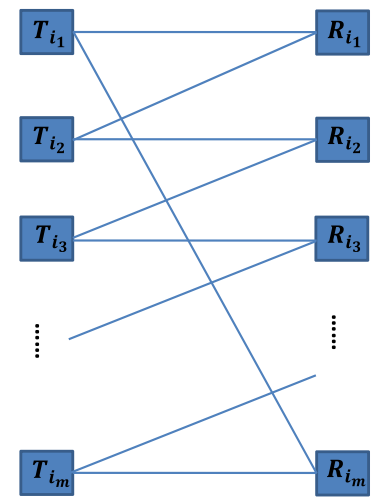

Fig. 8. The $m$-user cyclic interference channel.

(iii) The set $\mathcal{S}_{\overline{\mathbf{r}}}$ is bounded from below. Then, according to the fixed point theorem in [17] ([17, Proposition 6]), we know that for each $\mathbf{r} \in \mathcal{S}_{\overline{\mathbf{r}}}$, there exists a $\overline{\mathbf{r}} \leq \mathbf{r}$ such that $\overline{\mathbf{r}}=f(\overline{\mathbf{r}})$. Combining this result with (15), we know when (15)-(18) hold, there exist such $\bar{r}_{i}$ 's satisfying (53) and (54), which implies that $\left(d_{1}, d_{2}, \ldots, d_{K}\right) \in \mathcal{P}$. Therefore, we establish that $\mathcal{P}=\overline{\mathcal{P}}$.

\section{APPENDIX C \\ PROOF OF THEOREM 3}

First, each individual bound in (28) is simply the cut-set upper bound for user $i \in[K]$. Next, we consider the cyclic bound (29), where the modulo- $m$ arithmetic is implicitly used on the user indices, e.g., $i_{m}=i_{0}$. For any cyclic sequence $\left(i_{1}, i_{2}, \ldots, i_{m}\right) \in \Pi_{K}$, we start with the fully connected $K$-user interference channel with input-output relationship (1), and go through the following steps:

- Eliminate all the users $i \in[K] \backslash\left\{i_{1}, i_{2}, \ldots, i_{m}\right\}$ and their desired messages;

- Remove all the interfering links but the links from transmitter $i_{j}$ to receiver $i_{j-1}, \forall j \in\{1,2, \ldots, m\}$.

We end up with the $m$-user cyclic interference channel as depicted in Fig. 8. The above two steps cannot hurt the rates of the remaining messages. Therefore, the sum rate of users $i \in\left\{i_{1}, i_{2}, \ldots, i_{m}\right\}$ in the original $K$-user interference channel is upper bounded by that of the $m$-user cyclic interference channel. Define

$$
S_{i_{j}}(t)=h_{i_{j-1} i_{j}} \tilde{X}_{i_{j}}(t)+Z_{i_{j-1}}(t), \quad \forall j \in\{1,2, \ldots, m\} .
$$

Then for receiver $i_{j}$, we provide $S_{i_{j}}^{n}$ through a genie. From Fano's inequality, we have

$$
\begin{aligned}
& n\left(R_{i_{j}}-\epsilon\right) \\
& \leq I\left(W_{i_{j}} ; Y_{i_{j}}^{n}, S_{i_{j}}^{n}\right) \\
& =h\left(Y_{i_{j}}^{n}, S_{i_{j}}^{n}\right)-h\left(Y_{i_{j}}^{n}, S_{i_{j}}^{n} \mid W_{i_{j}}\right) \\
& =h\left(S_{i_{j}}^{n}\right)+h\left(Y_{i_{j}}^{n} \mid S_{i_{j}}^{n}\right)-h\left(S_{i_{j}}^{n} \mid W_{i_{j}}\right)-h\left(Y_{i_{j}}^{n} \mid S_{i_{j}}^{n}, W_{i_{j}}\right) \\
& =h\left(S_{i_{j}}^{n}\right)+h\left(Y_{i_{j}}^{n} \mid S_{i_{j}}^{n}\right)-h\left(Z_{i_{j-1}}^{n}\right)-h\left(S_{i_{j+1}}^{n}\right) .
\end{aligned}
$$

Taking the sum of $n\left(R_{i_{j}}-\epsilon\right)$ for all $j \in\{1,2, \ldots, m\}$, we obtain

$$
\begin{aligned}
n \sum_{j=1}^{m}\left(R_{i_{j}}-\epsilon\right) & \leq \sum_{j=1}^{m}\left[h\left(Y_{i_{j}}^{n} \mid S_{i_{j}}^{n}\right)-h\left(Z_{i_{j}}^{n}\right)\right] \\
& \leq \sum_{t=1}^{n} \sum_{j=1}^{m}\left[h\left(Y_{i_{j}}(t) \mid S_{i_{j}}(t)\right)-h\left(Z_{i_{j}}(t)\right)\right],
\end{aligned}
$$

where the last inequality follows chain rule and the fact that dropping conditioning does not reduce entropy. Finally, using the fact that the circularly symmetric Gaussian distribution maximizes conditional differential entropy under a given covariance constraint, we end up with the desired outer bound

$$
\sum_{j=1}^{m} R_{i_{j}} \leq \sum_{j=1}^{m} \log \left(1+\left|h_{i_{j} i_{j+1}}\right|^{2} P_{i_{j+1}}+\frac{\left|h_{i_{j} i_{j}}\right|^{2} P_{i_{j}}}{1+\left|h_{i_{j-1} i_{j}}\right|^{2} P_{i_{j}}}\right) .
$$

\section{APPENDIX D \\ Proof of Theorem 5}

We prove the theorem in two steps.

- Step 1: $\bigcup_{\mathcal{S} \subseteq[K]} \mathcal{P}_{\mathcal{S}} \subseteq \mathcal{P}^{*}$. It suffices to show that for all $\mathcal{S} \subseteq[K], \mathcal{P}_{\mathcal{S}} \subseteq \mathcal{P}^{*}$; i.e., the region $\mathcal{P}_{\mathcal{S}}$ can be achieved through TIN. Note that if $\mathcal{S}=\emptyset$, then $\mathcal{P}_{\mathcal{S}}=\mathcal{P}_{\emptyset}=\mathcal{P} \subseteq \mathcal{P}^{*}$.

Now, if $\mathcal{S} \neq \varnothing$, then to make the users in $\mathcal{S}$ silent, we set $r_{i}=-\infty, \forall i \in \mathcal{S}$. This forces $d_{i}=0$, $\forall i \in \mathcal{S}$. Then, for the remaining users, i.e., the users in $\mathcal{S}^{c}$, we use polyhedral TIN. Therefore, the polyhedral TIN region where all the users in $\mathcal{S}$ are removed from the network, can be achieved. This region is in fact $\mathcal{P}_{\mathcal{S}}$, and hence, $\mathcal{P}_{\mathcal{S}} \subseteq \mathcal{P}^{*}$.

- Step 2: $\mathcal{P}^{*} \subseteq \bigcup_{\mathcal{S} \subseteq[K]} \mathcal{P}_{\mathcal{S}}$. To prove this, we first define the sets $\tilde{\mathcal{P}}_{\mathcal{S}}$ as $\mathcal{P}_{\mathcal{S}}$ restricted to strictly positive GDoF's for users in $\mathcal{S}^{c}$; i.e.,

$$
\tilde{\mathcal{P}}_{\mathcal{S}}=\left\{\left(d_{1}, d_{2}, \ldots, d_{K}\right) \in \mathcal{P}_{\mathcal{S}}: d_{i}>0, \forall i \in \mathcal{S}^{c}\right\},
$$

for any $\mathcal{S} \subseteq[K]$. It is obvious that $\tilde{\mathcal{P}}_{\mathcal{S}} \subseteq \mathcal{P}_{\mathcal{S}}$ and therefore,

$$
\bigcup_{\mathcal{S} \subseteq[K]} \tilde{\mathcal{P}}_{\mathcal{S}} \subseteq \bigcup_{\mathcal{S} \subseteq[K]} \mathcal{P}_{\mathcal{S}}
$$

Now, we will prove that $\mathcal{P}^{*} \subseteq \bigcup_{\mathcal{S} \subseteq[K]} \tilde{\mathcal{P}}_{\mathcal{S}}$. Assume there exists a GDoF point $\left(d_{1}, d_{2}, \ldots, d_{K}\right)$ lying outside all of the sets $\tilde{\mathcal{P}}_{\mathcal{S}}$. Such a point should satisfy at least one of the following conditions:

- $d_{i}<0$ or $d_{i}>\alpha_{i i}$ for some user $i \in[K]$. In this case, it is trivial that the GDoF point is not achievable by TIN.

- $\sum_{j=1}^{m} d_{i_{j}}>\sum_{j=1}^{m}\left(\alpha_{i_{j} i_{j}}-\alpha_{i_{j-1} i_{j}}\right)$ for some cyclic sequence $\left(i_{1}, i_{2}, \ldots, i_{m}\right) \in \Pi_{K}$ such that $d_{i_{j}}>0$, $\forall j \in\{1,2, \ldots, m\}$. Note the modulo- $m$ arithmetic is implicitly used on the user indices, e.g., $i_{m}=i_{0}$. In this case, we show that this GDoF point cannot belong to $\mathcal{P}^{*}$ by contradiction. Assume otherwise; i.e., suppose $\left(d_{1}, d_{2}, \ldots, d_{K}\right)$ is achievable by TIN. 


$$
\begin{gathered}
\sum_{j=1}^{m} r_{i_{j}}+\alpha_{i_{j} i_{j}}-\max \left\{0, \max _{i_{k} \neq i_{j}}\left(r_{i_{k}}+\alpha_{i_{j} i_{k}}\right)\right\}>\sum_{j=1}^{m}\left(\alpha_{i_{j} i_{j}}-\alpha_{i_{j-1} i_{j}}\right) \\
\Rightarrow \sum_{j=1}^{m} r_{i_{j}}+\alpha_{i_{j-1} i_{j}}-\max \left\{0, \max _{i_{k} \neq i_{j}}\left(r_{i_{k}}+\alpha_{i_{j} i_{k}}\right)\right\}>0 .
\end{gathered}
$$

For all $j \in\{1,2, \ldots, m\}$, since it is assumed that $d_{i_{j}}>0$, there exist $r_{i_{j}}$ 's such that

$$
d_{i_{j}}=r_{i_{j}}+\alpha_{i_{j} i_{j}}-\max \left\{0, \max _{i_{k} \neq i_{j}}\left(r_{i_{k}}+\alpha_{i_{j} i_{k}}\right)\right\} .
$$

Therefore, we will have (58) at the top of the page. On the other hand, for all $j \in\{1,2, \ldots, m\}$ we have

$$
\max \left\{0, \max _{i_{k} \neq i_{j}}\left(r_{i_{k}}+\alpha_{i_{j} i_{k}}\right)\right\} \geq r_{i_{j+1}}+\alpha_{i_{j} i_{j+1}},
$$

which in turn implies that

$$
\begin{aligned}
& \sum_{j=1}^{m} r_{i_{j}}+\alpha_{i_{j-1} i_{j}}-\max \left\{0, \max _{i_{k} \neq i_{j}}\left(r_{i_{k}}+\alpha_{i_{j} i_{k}}\right)\right\} \\
& \leq \sum_{j=1}^{m} r_{i_{j}}+\alpha_{i_{j-1} i_{j}}-\left(r_{i_{j+1}}+\alpha_{i_{j} i_{j+1}}\right) \\
& \quad=\sum_{j=1}^{m}\left(r_{i_{j}}-r_{i_{j+1}}\right)+\sum_{j=1}^{m}\left(\alpha_{i_{j-1} i_{j}}-\alpha_{i_{j} i_{j+1}}\right) \\
& \quad=0 .
\end{aligned}
$$

But considering (58), this is a contradiction. Therefore in this case, the GDoF point is not achievable by TIN, too.

This implies that $\mathcal{P}^{*} \subseteq \bigcup_{\mathcal{S} \subseteq[K]} \tilde{\mathcal{P}}_{\mathcal{S}}$, which combined with (57) yields $\mathcal{P}^{*} \subseteq \bigcup_{\mathcal{S} \subseteq[K]} \mathcal{P}_{\mathcal{S}}$.

Combining steps 1 and 2 leads to (42), therefore completing the proof.

\section{ACKNOWLEDGMENT}

The authors would like to thank the Associate Editor, and the reviewers for their valuable comments.

\section{REFERENCES}

[1] C. Geng, N. Naderializadeh, A. S. Avestimehr, and S. A. Jafar, "On the optimality of treating interference as noise," in Proc. 51st Annu. Allerton Conf. Commun., Control, Comput., Oct. 2013, pp. 1166-1173.

[2] M. A. Charafeddine, A. Sezgin, Z. Han, and A. Paulraj, "Achievable and crystallized rate regions of the interference channel with interference as noise," IEEE Trans. Wireless Commun., vol. 11, no. 3, pp. 1100-1111, Mar. 2012.

[3] C. W. Tan, M. Chiang, and R. Srikant, "Fast algorithms and performance bounds for sum rate maximization in wireless networks," IEEE/ACM Trans. Netw., vol. 21, no. 3, pp. 706-719, Jun. 2013.

[4] A. S. Motahari and A. K. Khandani, "Capacity bounds for the Gaussian interference channel," IEEE Trans. Inf. Theory, vol. 55, no. 2, pp. 620-643, Feb. 2009.

[5] V. S. Annapureddy and V. V. Veeravalli, "Gaussian interference networks: Sum capacity in the low-interference regime and new outer bounds on the capacity region," IEEE Trans. Inf. Theory, vol. 55, no. 7, pp. 3032-3050, Jul. 2009.

[6] X. Shang, G. Kramer, and B. Chen, "A new outer bound and the noisyinterference sum-rate capacity for Gaussian interference channels," IEEE Trans. Inf. Theory, vol. 55, no. 2, pp. 689-699, Feb. 2009.
[7] V. S. Annapureddy and V. V. Veeravalli, "Sum capacity of MIMO interference channels in the low interference regime," IEEE Trans. Inf. Theory, vol. 57, no. 5, pp. 2565-2581, May 2011.

[8] R. H. Etkin, D. N. C. Tse, and H. Wang, "Gaussian interference channel capacity to within one bit," IEEE Trans. Inf. Theory, vol. 54, no. 12, pp. 5534-5562, Dec. 2008.

[9] S. A. Jafar and S. Vishwanath, "Generalized degrees of freedom of the symmetric Gaussian $K$ user interference channel," IEEE Trans. Inf. Theory, vol. 56, no. 7, pp. 3297-3303, Jul. 2010.

[10] L. Zhou and W. Yu, "On the capacity of the $K$-user cyclic Gaussian interference channel," IEEE Trans. Inf. Theory, vol. 59, no. 1, pp. 154-165, Jan. 2013.

[11] J. Zander and M. Frodigh, "Comment on "performance of optimum transmitter power control in cellular radio systems," IEEE Trans. Veh. Technol., vol. 43, no. 3, p. 636, Aug. 1994.

[12] A. Schrijver, Combinatorial Optimization. New York, NY, USA: Springer-Verlag, 2003.

[13] V. Erceg et al., "An empirically based path loss model for wireless channels in suburban environments," IEEE J. Sel. Areas Commun., vol. 17, no. 7, pp. 1205-1211, Jul. 1999.

[14] N. Naderializadeh and A. S. Avestimehr, "ITLinQ: A new approach for spectrum sharing in device-to-device communication systems," IEEE J. Sel. Areas Commun., vol. 32, no. 6, pp. 1139-1151, Jun. 2014.

[15] Y. Zhao, C. W. Tan, A. S. Avestimehr, S. N. Diggavi, and G. J. Pottie, "On the maximum achievable sum-rate with successive decoding in interference channels," IEEE Trans. Inf. Theory, vol. 58, no. 6, pp. 3798-3820, Jun. 2012.

[16] Z. Shao, M. Chen, A. S. Avestimehr, and S.-Y. R. Li, "Cross-layer optimization for wireless networks with deterministic channel models," IEEE Trans. Inf. Theory, vol. 57, no. 9, pp. 5840-5862, Sep. 2011.

[17] H. Persson, "A fixed point theorem for monotone functions," Appl. Math. Lett., vol. 19, no. 11, pp. 1207-1209, Nov. 2006.

Chunhua Geng received the B.E. degree in Communications Engineering from Beijing Jiaotong University, Beijing, China, in 2007, and the M.S. degree in Electronic Engineering from Tsinghua University, Beijing, China, in 2010. He is currently pursuing the Ph.D. degree in the Department of Electrical Engineering and Computer Science, University of California, Irvine. His research interests include network information theory, communication theory, information theoretical security, and wireless communications.

Navid Naderializadeh received his B.S. degree in electrical engineering from Sharif University of Technology, Tehran, Iran, in 2011 and his M.S. degree in electrical and computer engineering from Cornell University, Ithaca, NY, in 2014. He joined University of Southern California, Los Angeles, CA, in 2014, where he is pursuing his Ph.D. in the department of electrical engineering. His research is focused on developing spectrum sharing mechanisms in wireless networks, especially in device-to-device (D2D) communication networks. Navid obtained the first rank in the Iranian nationwide university entrance exam in 2007. He was also a recipient of Jacobs scholarship in 2011. 
Amir Salman Avestimehr received the B.S. degree in electrical engineering from Sharif University of Technology, Tehran, Iran, in 2003 and the M.S. degree and Ph.D. degree in electrical engineering and computer science, both from the University of California, Berkeley, in 2005 and 2008, respectively. $\mathrm{He}$ is currently an Associate Professor at the EE department of University of Southern California, Los Angeles, CA. He was also a postdoctoral scholar at the Center for the Mathematics of Information (CMI) at the California Institute of Technology, Pasadena, in 2008. His research interests include information theory, the theory of communications, and their applications.

Dr. Avestimehr has received a number of awards for research and teaching, including the Communications Society and Information Theory Society Joint Paper Award in 2013, the Presidential Early Career Award for Scientists and Engineers (PECASE) in 2011, the Michael Tien 72 Excellence in Teaching Award in 2012, the Young Investigator Program (YIP) award from the U.S. Air Force Office of Scientific Research in 2011, the National Science Foundation CAREER award in 2010, and the David J. Sakrison Memorial Prize in 2008. He is currently an Associate Editor for the IEEE TRANSACTIONS ON INFORMATION THEORY. He has also been a Guest Associate Editor for the IEEE TRANSACTIONS ON INFORMATION THEORY Special Issue on Interference Networks and General Co-Chair of the 2012 North America Information Theory Summer School and the 2012 Workshop on Interference Networks.
Syed A. Jafar (S'99-M'04-SM'09-F'14) received his B. Tech. from IIT Delhi, India, in 1997, M.S. from Caltech, USA, in 1999, and Ph.D. from Stanford, USA, in 2003, all in Electrical Engineering. His industry experience includes positions at Lucent Bell Labs, Qualcomm Inc. and Hughes Software Systems. He is a Professor in the Department of Electrical Engineering and Computer Science at the University of California Irvine, Irvine, CA USA. His research interests include multiuser information theory, wireless communications and network coding.

Dr. Jafar received the NSF CAREER award in 2006, the ONR Young Investigator Award in 2008, and the School of Engineering Maseeh Outstanding Research Award in 2010, his co-authored papers received Best Journal Paper Awards from the IEEE Information Theory Society in 2009, from the IEEE Communication Society in 2013, and from the IEEE Signal Processing Society in 2015, and Best Conference Paper Awards at IEEE GLOBECOM 2012 and at IEEE GLOBECOM 2014 Dr. Jafar received the UC Irvine EECS Professor of the Year award five times, in 2006, 2009, 2011, 2012, and 2014, from the Engineering Students Council and the Teaching Excellence Award in 2012 from the School of Engineering. He was a University of Canterbury Erskine Fellow in 2010 and an IEEE Communications Society Distinguished Lecturer for 2013-2014. Dr. Jafar was recognized as a Thomson Reuters Highly Cited Researcher and included by Sciencewatch among The World's Most Influential Scientific Minds in 2014. He served as Associate Editor for IEEE TRANSACTIONS ON COMMUNICATIONS 2004-2009, for IEEE COMMUNICATIONS LETTERS 2008-2009 and for IEEE TRANSACTIONS ON INFORMATION THEORY 2009-2012. Dr. Jafar was elevated to IEEE Fellow, Class of 2014, for contributions to analyzing the capacity of wireless communication networks. 